Research Article

\title{
Study and Analysis of a Multilayer Multipair Electret-Based Thin-Film Mechanical Antenna
}

\author{
K. P. Zhou $\mathbb{D}^{1}{ }^{1}$ Y. T. Niu $\mathbb{D}^{1,},{ }^{1,2}$ W. N. Liu, ${ }^{1,2}$ Z. D. Wang, ${ }^{1}$ S. H. Guo, ${ }^{1}$ B. Li, ${ }^{1}$ Z. Wang, \\ and X. K. Zhao ${ }^{1}$ \\ ${ }^{1}$ College of Electronic and Electrical Engineering, Henan Normal University, Xinxiang 453000, China \\ ${ }^{2}$ Henan Electromagnetic Wave Academician Workstation, Xinxiang 453000, China \\ Correspondence should be addressed to Y. T. Niu; 970131594@qq.com
}

Received 19 July 2021; Revised 2 November 2021; Accepted 5 November 2021; Published 26 November 2021

Academic Editor: Stefano Selleri

Copyright (c) 2021 K. P. Zhou et al. This is an open access article distributed under the Creative Commons Attribution License, which permits unrestricted use, distribution, and reproduction in any medium, provided the original work is properly cited.

\begin{abstract}
Very low-frequency (VLF) and ultralow-frequency (ULF) electromagnetic waves have the advantage of high penetration and low propagation loss in wireless communication systems and are mainly used for underwater and underground communications, as well as for earthquake and lightning forecasting. At present, VLF and ULF antennas are mostly bulky and require hundreds of antennas and more to be set up, which is costly and inefficient. In this paper, we propose to generate VLF and ULF signals by rotating a multilayer multipair electret thin-film electret driven by an excitation device, which improves the problem of low radiation efficiency of VLF and ULF signals and the large size of conventional low-frequency transmitting antennas. Based on a multilayer, multipair electret film mechanical antenna, a magnetic field propagation model is developed, and the relationship between the magnetic flux density mode and the number of layers of electret films, as well as the relationship between the antenna emission frequency and the motor rotation frequency and the number of pairs of electret films, is analyzed. The selection of a suitable model for practical situations based on conditions such as antenna size and propagation distance is illustrated. The research work is of great importance for guiding the design of mechanical antennas and optimizing antenna structures.
\end{abstract}

\section{Introduction}

High-frequency wireless communication has the advantages of large bandwidth, fast communication rate, and low latency; compared to high-frequency wireless communication, the low-frequency electromagnetic wave has the characteristics of high communication reliability, strong penetration, and longer communication distance. When electromagnetic waves are propagated in other media such as water and underground, high-frequency electromagnetic waves will attenuate very quickly, resulting in poor communication quality in other media such as underwater and underground, while very low-frequency and ultralow-frequency electromagnetic waves can propagate over longer distances in other media such as underwater and underground due to their excellent penetration [1].

The size of the low-frequency transmitting antennas currently used in the world is extremely large; for example, a wireless transmitter operating at $300 \mathrm{~Hz}$ requires an antenna equivalent to one-eighth to one-half the length of the wavelength, i.e., 100 to $500 \mathrm{~km}$ long. As a result, existing ultralow-frequency communication devices are expensive, have large systems, and are characterized by high power consumption. To address the shortcomings of conventional low-frequency transmitting antennas, such as their large size and low efficiency, in 2017, the US Department of Defense Advanced Research Projects Agency (DARPA) proposed the (AMEBA) program, which aims to develop a portable low-frequency wireless transmission system capable of generating carriers below $30 \mathrm{kHz}$ [2]. Once developed, this technology could enable ground-penetrating or underwater communications in mines and on the ground in the civilian sector; in the military sector, VLF and ULF communications could enable communication between submarines. After the AMEBA project was proposed, a 
lot of research was started at home and abroad. So far, both domestic and international research on mechanical antennas has made some progress. Reference [3] proposed a VLF (Very Low-Frequency) transmitter based on a piezoelectric crystal, the scheme used adds AC to the two ends of the piezoelectric crystal, the piezoelectric crystal is machined into a quadrilateral shape, when an electrical signal is added to the two ends of the crystal, the crystal will stretch and deform according to the law of the added electrical signal, the reciprocal stretch and deformation belong to mechanical vibration, and the mechanical vibration of the piezoelectric crystal will generate a changing electromagnetic field around it [3]. In [4], the team of Cui Yong and Wang Chen invented the patent of "A rotating electret-type mechanical antenna low-frequency communication system" in 2018, including a low-frequency electromagnetic signal generation module and a magnetic signal reception module, in which the transmitter adopts a rotating electret scheme; in [5], the low-frequency magnetic field is excited by the changing electric field by rotating parallel placed, differently charged pole plates to cause relative motion. In [6], a new modulation method-polarization modulation-is proposed based on a rotating permanent magnet type mechanical antenna, which avoids the high load on the motor caused by frequent adjustment of the motor frequency; in addition, it is proposed in [7] that, from a macroscopic analysis, the effect of rotating a uniformly magnetized permanent magnet is equivalent to applying a current to its surface, and a model based on this theory is established and relevant theoretical calculations and formulae are derived.

Mechanical antenna technology offers the possibility of miniaturization of low-frequency antennas; however, most of the current research is focused on permanent magnet mechanical antennas; compared to permanent magnet mechanical antennas, electret mechanical antennas have a slower decay rate, and with the increasing maturity of organic electret technology, mechanical antennas based on flexible electret materials have the advantages of a lightweight, high plasticity, and controllable charge compared to permanent magnet mechanical antennas. The advantages of electret materials are as follows. As the name suggests, an electret is an object that remains in a polarised state under external excitation and is also known as a permanent electrode [8-10]. The increasing maturity of electret material fabrication technology has brought greater possibilities for the research of electret-type mechanical antennas $[11,12]$. At present, electret films can be made below the millimeter level and have a high charge storage capacity, which can effectively reduce the size of low-frequency transmitting antennas. The number of layers of the electret film also has an impact on the radiation intensity of the mechanical antenna, and the amplitude can be adjusted by increasing the number of layers of the electret film; the use of electret film type mechanical antenna can effectively reduce the size of the low-frequency transmitting antenna and, at the same time, make the mechanical antenna designed as a wearable device. The use of electret film mechanical antennas can effectively reduce the size of low-frequency transmitting antennas, while making the mechanical antennas designed as wearable devices, bringing great convenience to the staff concerned. In summary, changing the charge distribution pattern by varying the number of layers and pairs of electret films brings great possibilities to change the radiation frequency and increase the propagation distance of mechanical antennas, which brings great possibilities to the research of mine communication, submarine communication, and wearable low-frequency transmitting antennas. Moreover, the research on electret mechanical antennas is relatively small and belongs to a new field. At the same time, since low-frequency communication is of great significance to military fields such as timing and navigation systems, this paper is a study of the multilayer multipair electret mechanical antenna. Therefore, in this paper, the characteristics of multilayer multipair electret thin-film mechanical antennas are studied and analyzed.

\section{Two-Dimensional Magnetic Field Propagation Model for Electret-Type Mechanical Antennas}

The basic structure of an electret mechanical antenna is shown in Figure 1, where an electret film with a heterogeneous charge is symmetrically attached to the antenna housing and a low permeability material is used for the antenna support to ensure the normal passage of the magnetic field signal in the multilayer electret film. When the motor drives the cylindrical structure, the electret film rotates to produce two toroidal currents in opposite directions, so that the changing electric field excites a changing magnetic field [13]. For the entire cylindrical electret film, the magnetic field generated can be calculated by superimposing the magnetic field generated by each of the planar rings. A two-dimensional planar torus is shown in Figure 2, assuming that the electret film is thin enough to neglect its thickness and that the entire cylindrical support is uncharged.

According to the Biot-Safar law [13], the magnetic inductance generated by the current microelement in each transmitting antenna is

$$
B_{\text {mic }}=\left|\vec{B}_{\text {mic }}\right|=\left|\frac{\mu_{0}}{4 \pi} \frac{I \cdot \Delta \vec{s} \times \vec{l}}{D^{2}}\right|,
$$

where $\mu_{0}$ is the magnetic permeability, $I$ is the current through the microelement, $\Delta \vec{s}$ is the length of the microelement, and $\vec{l}$ is $D$ the unit direction vector. 


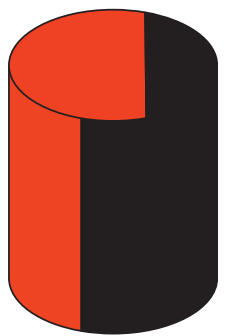

FIGURE 1: Basic structure of an electret thin-film mechanical antenna.

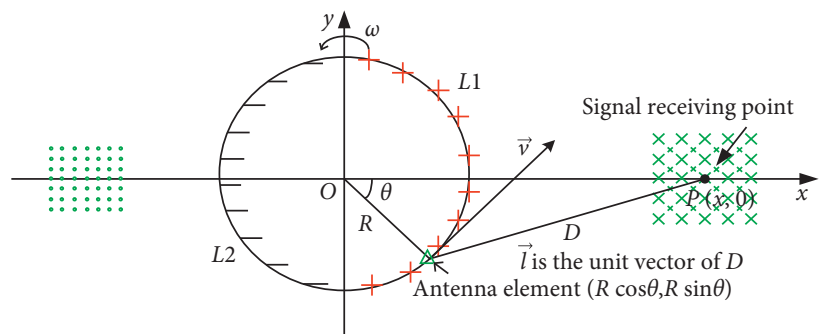

FIgURE 2: Principle of two-dimensional magnetic field propagation in an electret thin-film mechanical antenna.

According to the relationship between current and charge, the relationship between current and moving charge is

$$
I \Delta \vec{s}=n q S \vec{v} \Delta s,
$$

where $n$ is the number of charges, $S$ is the cross-sectional area of the microelement, $q$ is the amount of charge, $v$ is the velocity vector of charge motion, and the charge density is $\sigma=n q S$.

From the relationship between current $I$ and charge density, the magnetic induction generated by each current microelement can be calculated as

$$
B_{\text {mic }}=\left|\vec{B}_{\text {mic }}\right|=\left|\frac{\mu_{0} \sigma}{4 \pi} \frac{\vec{v} \times \vec{l}}{D^{2}} \Delta \Delta s\right| .
$$

Loop integration of the magnetic induction microelement gives

$$
B=|\vec{B}|=\left|\lim _{\Delta s \longrightarrow 0} \sum \vec{B}_{\mathrm{mic}}\right|=\left|\oint \frac{\mu_{0} \sigma}{4 \pi} \frac{\vec{v} \times \vec{l}}{D^{2}} \Delta \Delta s\right| .
$$

When ignoring the size of the transmitting antenna and assuming that $D$ is equal to the distance to the origin $x$, using the symmetry theorem, we get

$$
\begin{aligned}
B & =B_{n}+B_{n}=2 B_{n}=2\left|\int_{-\pi / 2}^{\pi / 2} \frac{\mu_{0} \sigma}{4 \pi} \frac{\vec{v} \times \vec{l}}{D^{2}} R \mathrm{~d} \theta\right| \\
& =\frac{\mu_{0} \sigma \omega R^{2}}{2 \pi x^{2}} \int_{-\pi / 2}^{\pi / 2} \cos \theta \mathrm{d} \theta \\
& =\frac{\mu_{0} \sigma \omega R^{2}}{\pi x^{2}} .
\end{aligned}
$$

When the antenna is turned by a certain angle, the magnetic fields shaded in Figure 3 on the $x$-axis will cancel each other out, so equation (5) can be converted into (where $\omega$ is the angular velocity of the rotation of the circle, and $t$ is the rotation time)

$$
\begin{aligned}
B & =2 B_{n}=2\left|\int_{-\pi / 2+\omega t}^{\pi / 2-\omega t} \frac{\mu_{0} \sigma}{4 \pi} \cdot \frac{\vec{v} \times \vec{l}}{D^{2}} R \mathrm{~d} \theta\right| \\
& =\frac{\mu_{0} \sigma \omega R^{2}}{2 \pi x^{2}} \int_{-\pi / 2+\omega t}^{\pi / 2-\omega t} \cos \theta \mathrm{d} \theta \\
& =\frac{\mu_{0} \sigma \omega R^{2}}{\pi x^{2}} \cos \omega t .
\end{aligned}
$$

If the receiving point of the signal is not $x$ on the axis but anywhere in the plane, $x-y$, the magnetic fields generated by the shaded part of the transmitting antenna in Figure 3 at the receiving point will cancel each other out, and now $\omega t=0$, so that equation (5) will be transformed into

$$
\begin{aligned}
B & =2\left|\int_{-\pi / 2}^{(\pi / 2)-2 \beta} \frac{\mu_{0} \sigma}{4 \pi} \cdot \frac{\vec{v} \times \vec{l}}{D^{2}} R \mathrm{~d} \theta\right|=\frac{\mu_{0} \sigma \omega R^{2}}{2 \pi x^{2}} \int_{-\pi / 2}^{(\pi / 2)-2 \beta} \cos \theta \mathrm{d} \theta \\
& =\frac{\mu_{0} \sigma \omega R^{2}}{2 \pi x^{2}}(\cos 2 \beta+1),
\end{aligned}
$$

Let $\cos \varphi=(\cos 2 \beta+1) / 2$; then, equation (7) becomes

$$
B=\frac{\mu_{0} \sigma \omega R^{2}}{\pi x^{2}} \cos \varphi
$$

Compared to the magnetic induction on the $x$ axis, the magnetic induction at any position increases only by the 


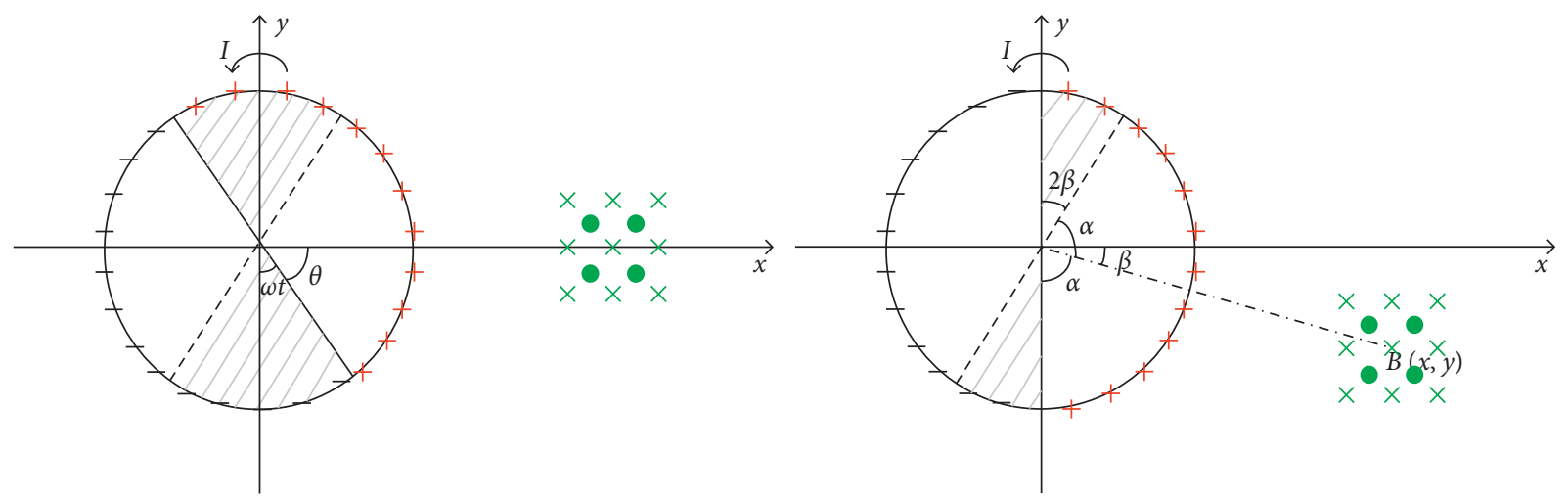

FIGURE 3: Receiving condition when the receiving point is on the $x$-axis; receiving condition when the receiving point is not on the $x$-axis.

initial phase angle $\varphi$, and the initial phase angle $x$ on the axis is $\varphi=0$.

Combining equations (5)-(8), the magnetic induction at any point in time in the plane is calculated as follows $x-y$.

$$
B=\frac{\mu_{0} \sigma \omega R^{2}}{\pi D^{2}} \cos (\omega t+\varphi),
$$

where $\beta=\arctan (y / x)$, and $\varphi=\arccos (\cos 2 \beta+1 / 2)$.

It can be seen from equation (9) that when ignoring the effect of antenna size, the magnetic $x-y$ induction vector mode at any point in the plane varies with time according to the cosine function, and the maximum value of the magnetic induction mode at the same distance to the source $D$ is the same. Therefore, it is easy to know $x-y$ that the direction of the normal of the equivalence line in the plane is the direction of the axis $x$.

In summary, the study of the $D$ variation of magnetic induction with distance can be reduced to the variation of the magnetic induction mode with $x$ the horizontal coordinate on the axis.

\section{Three-Dimensional Magnetic Field Propagation Model for Electret-Type Mechanical Antennas}

The magnetic field generated by a flat circular ring is integrated over the height (as shown in Figure 4) to obtain the magnetic field propagation model for a three-dimensional cylindrical antenna, which is discussed below for neglecting the transmitting antenna size and considering the transmitting size, respectively [13].

When ignoring the size of the transmitting antenna, for any signal reception point in space $P(x, y, z)$, it is known from equations (3)-(5) that

$$
\begin{aligned}
D & =\sqrt{x^{2}+y^{2}+z^{2}}, \\
\vec{v} & =(\omega R \sin \theta, \omega R \cos \theta, 0), \\
\vec{l} & =\left(\frac{x}{\sqrt{x^{2}+y^{2}+z^{2}}}, \frac{y}{\sqrt{x^{2}+y^{2}+z^{2}}}, \frac{z}{\sqrt{x^{2}+y^{2}+z^{2}}}\right), \\
\vec{v} \times \vec{l} & =\left(\begin{array}{l}
\frac{\omega R \sin \theta}{\sqrt{x^{2}+y^{2}+z^{2}}} \frac{j}{\sqrt{x^{2}+y^{2}+z^{2}}} \\
=\left(\frac{\omega z R \cos \theta}{\sqrt{x^{2}+y^{2}+z^{2}}}, \frac{-\omega z R \sin \theta}{\sqrt{x^{2}+y^{2}+z^{2}}}, \frac{\omega y R \sin \theta}{\sqrt{x^{2}+y^{2}+z^{2}}}-\frac{\omega x R \cos \theta}{\sqrt{x^{2}+y^{2}+z^{2}}}\right.
\end{array}\right),
\end{aligned}
$$




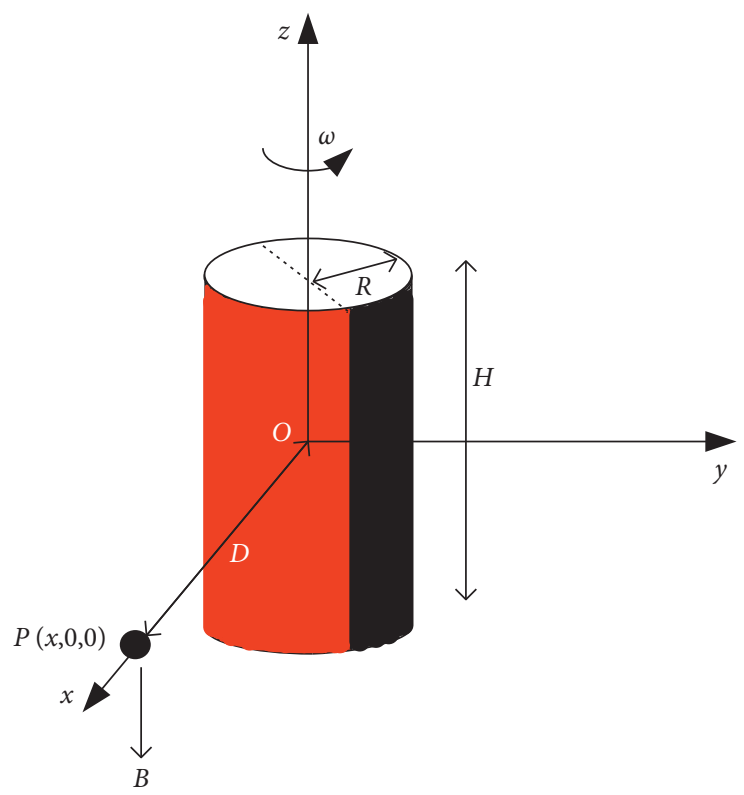

Figure 4: Schematic diagram of three-dimensional electret magnetic field propagation.

$$
B=|\vec{B}|=\left|2 \int_{-H / 2}^{H / 2} \int_{-\pi / 2}^{\pi / 2} \frac{\mu_{0} \sigma}{4 \pi} \cdot \frac{\vec{v} \times \vec{l}}{D^{2}} R \mathrm{~d} \theta\right|=\left|\frac{\mu_{0} \sigma H R}{2 \pi D^{2}} \int_{-\pi / 2+\omega t}^{\pi / 2-\omega t} \vec{v} \times \vec{l} \mathrm{~d} \theta\right|,
$$

where $H$ is the height of the cylinder, $\sigma$ is the charge density on the outer surface of the cylinder, and $R$ is the radius of the cylinder.

From equations (13) and (14), we can see that when the $z$ coordinates are 0 (i.e., when the signal reception point is in $x-y$ the plane), the components of the magnetic induction vector in both $y$ and $x$ directions are 0 , and the modulus of the magnetic induction is the magnitude of the component in the corresponding $z$ direction. Therefore, it is advantageous to simplify the problem and reduce the computational effort when the signal reception point is $x-y$ in the plane. Based on the discussion of the two-dimensional case in the previous section, it is found that the variation of the maximum value of the modulus of magnetic induction at different locations on the $x$ axis is the same as the variation of the maximum value of the modulus of magnetic induction at any point in space with distance $D$ (perpendicular to the equivalence plane), so the problem can be further simplified by discussing the variation of the maximum value of the modulus of magnetic induction at different locations on the axis $x$.

\section{Numerical Simulation of a Cylindrical Electret Mechanical Antenna}

COMSOL Multiphysics is a multiphysics finite element analysis software, used in the simulation of various engineering problems, including structural mechanics module (structural mechanics), RF module (RF), AC/DC module, micromotor module (MEMS), magnetic field module (magnetic field module), acoustics module (acoustics), and optical module. In this paper, the electret mechanical antenna simulation uses the 3D magnetic field module, which can do 2D and 3D simulations of low-frequency electric and magnetic fields [6].

\section{Simulation and Analysis of Single-Pair and Multipair Electret Thin-Film Mechanical Antennas}

Because the object of study is a low-frequency transmitting antenna, and the antenna transmitting frequency is related to the motor speed, we take the motor speed as $\omega=1000 \pi \mathrm{rad} / \mathrm{s}$; i.e., $f=500 \mathrm{~Hz}$.

According to data published by the US DARPA Bureau Mechanical Antenna Project [14], the electret surface charge density is taken, $\sigma=3400 \mathrm{n} \mathrm{C} / \mathrm{cm}^{2}$, the electret cylinder outer diameter is $R=1 \mathrm{~cm}$, the height is $H=3 \mathrm{~cm}$, and the magnetic permeability is set approximately equal to the magnetic permeability in a vacuum $\mu_{0}=4 \pi \times 10^{-7}$. Finally, a probe with an axis is added to the calculation area for extracting the variation of the magnetic field at a point with time and the variation of the magnetic induction intensity with distance.

For the sake of simplicity, this paper first simulates a single-layer, single-pair electret film mechanical antenna and then increases the number of layers and pairs of electret films in turn to investigate a multilayer, multipair electret film mechanical antenna.

Firstly, the geometric model of the mechanical antenna can be modeled as shown in Figure 5, Figure 6 shows a 3D model of a single-layer single-pair electret mechanical 


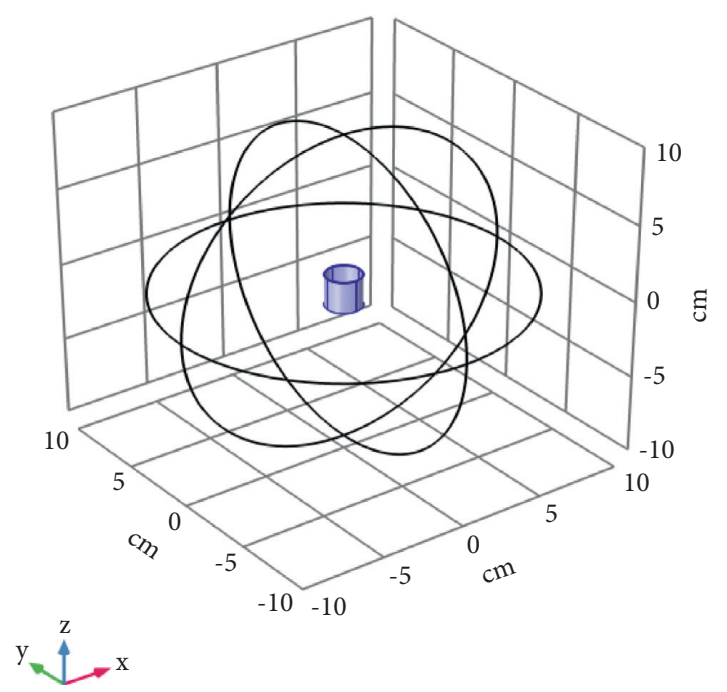

FIgURE 5: COMSOL 3D simulation diagram.

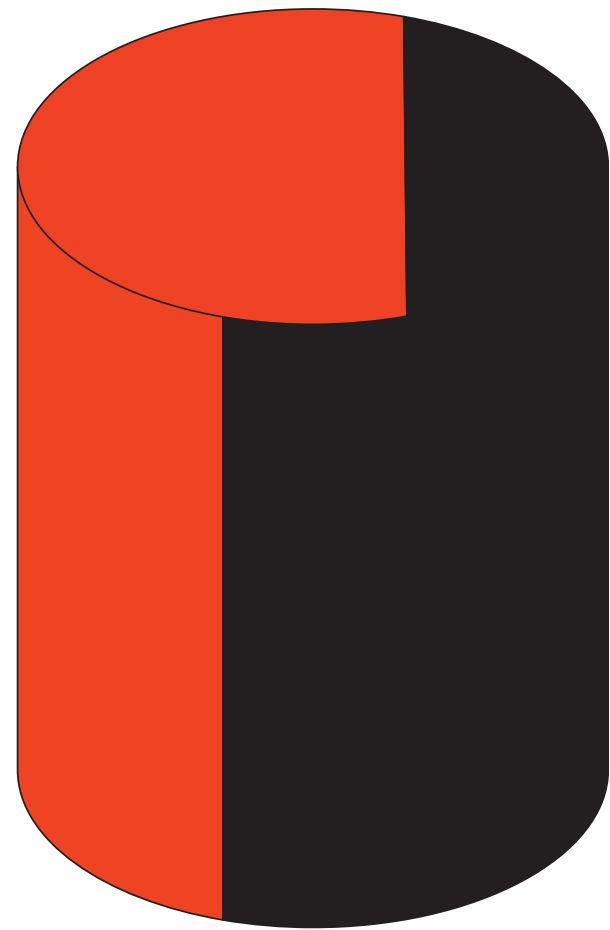

FIGURE 6: Structure of a single-layer single pair $(a=1, b=1)$ electret thin-film mechanical antenna.

antenna, and the single-layer single-pair electret thin-film mechanical antenna can be studied by introducing the parameters such as electret surface charge density, polarization mode, and magnetic permeability, and the magnetic field cloud can be obtained as shown in Figure 7.

Simulation of the data at the probe and the axis according to the parameters set in advance, the simulation results are the data set, and the data obtained at the probe and the axis can be imported into the Origin drawing

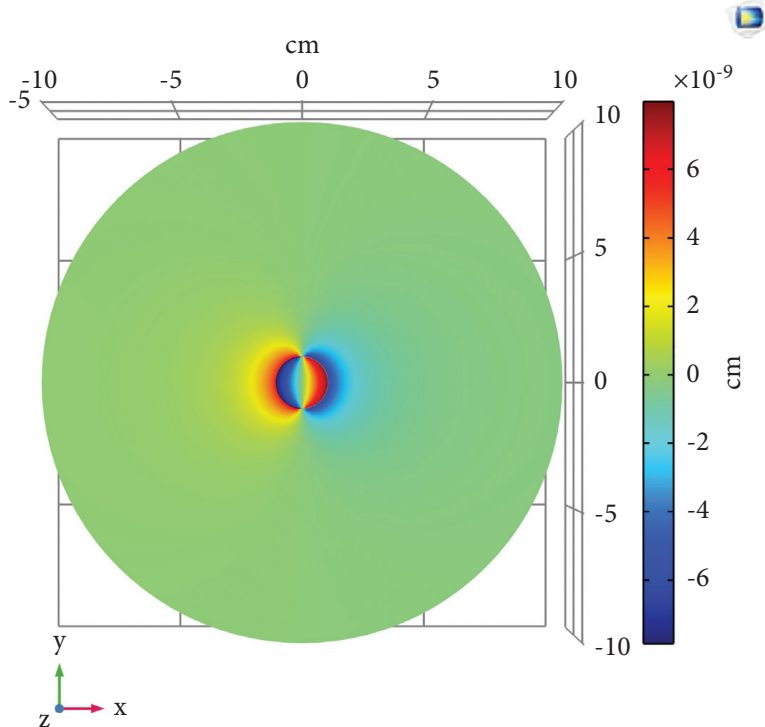

Figure 7: Magnetic field cloud at $a=1$ and $b=1$.

software to obtain the variation of the magnetic induction intensity at the probe with time and the variation of the magnetic induction intensity $B$ at the axis with distance $D$ for a single layer and a single pair. Though the calculation can be derived from the $S$ parameter changes as shown in Figure 8, a known electret-type mechanical antenna radiates circularly polarized waves, and setting the radiation field in the far area can obtain the $E$-plane gain and $\mathrm{H}$-plane gain as in Figures 9 and 10 .

According to the data obtained from the probe, the image of the change of magnetic induction intensity at the probe with time is drawn in Figure 11, from which it can be seen that the change of magnetic induction intensity at the probe with time is a sinusoidal regular periodic change; doing Fourier transform on the data in Figure 11, we can get 


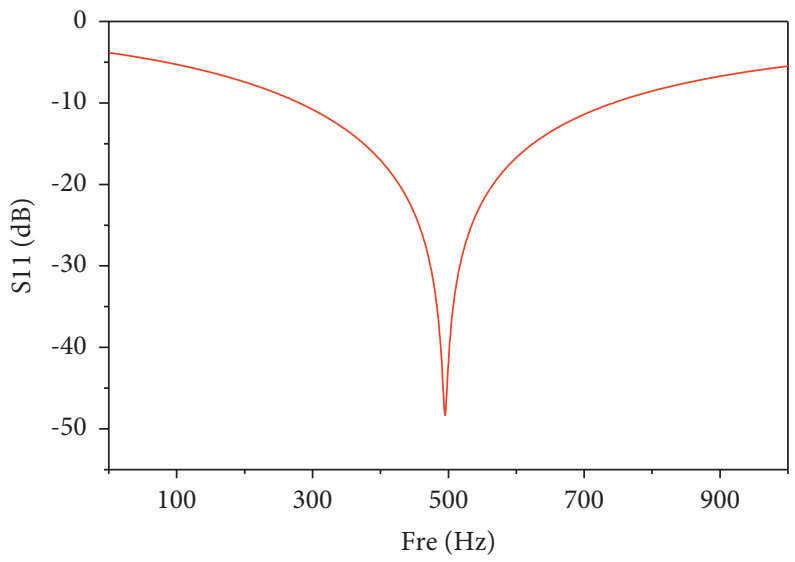

B

Figure 8: S11 parameters for $a=1$ and $b=1$.
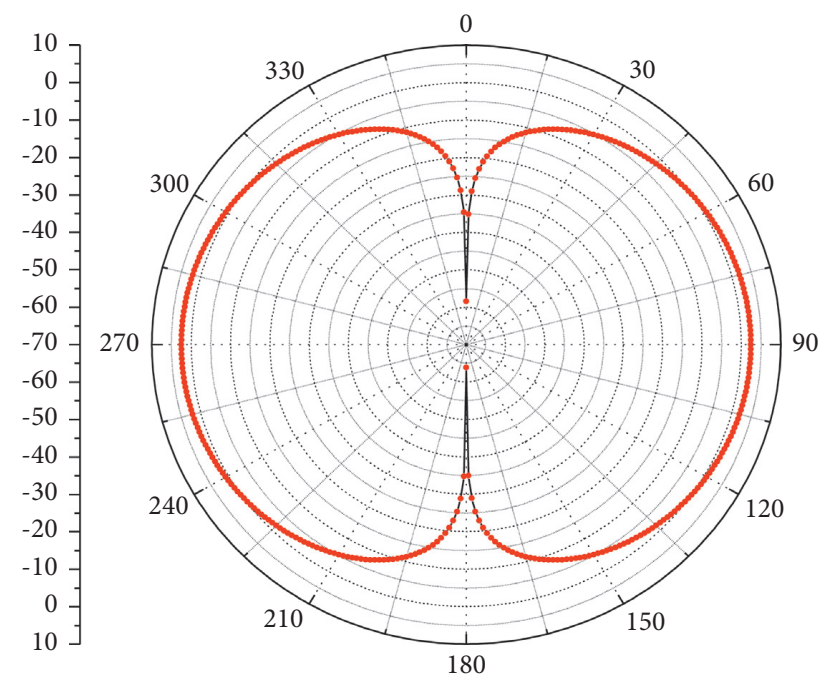

E-Gain

Figure 9: E-surface gain diagram for $a=1$ and $b=1$.

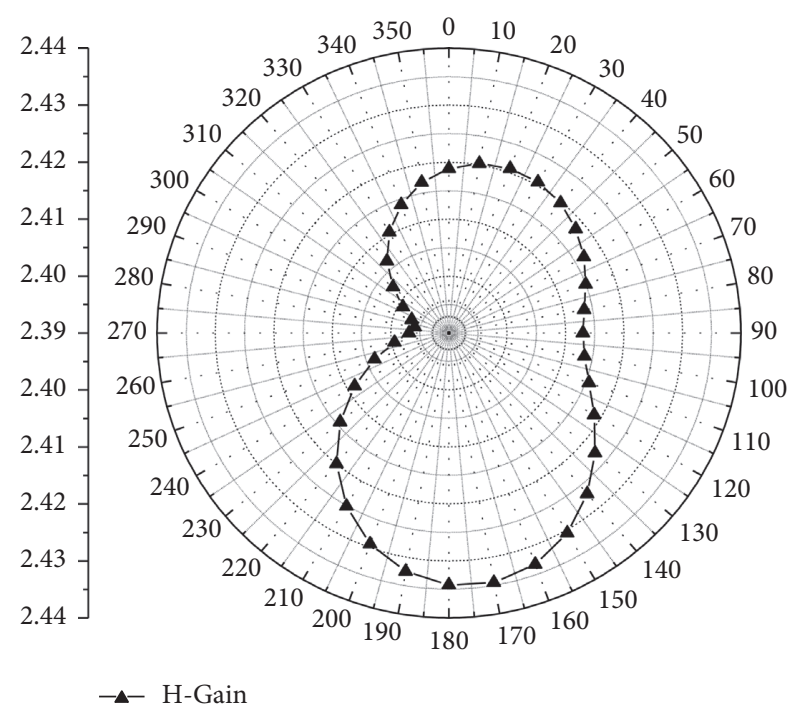

Figure 10: H-surface gain diagram for $a=1$ and $b=1$.

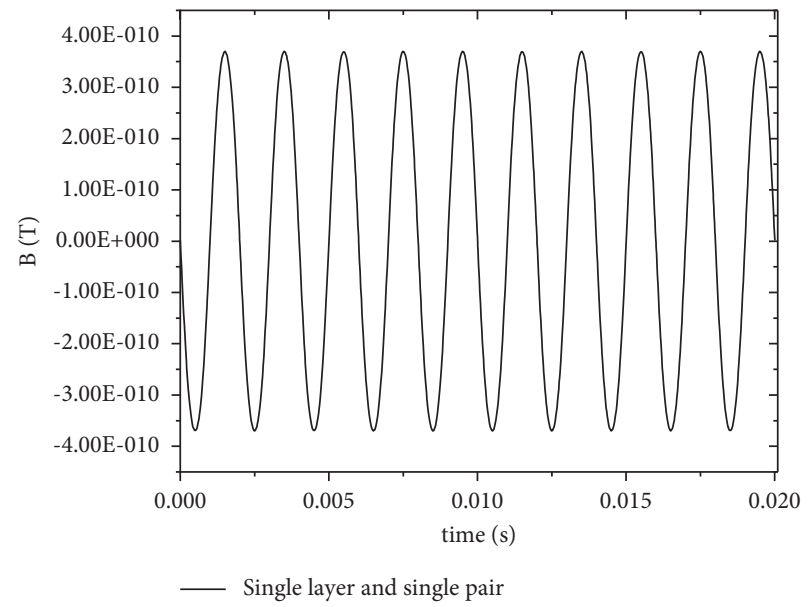

FIgURE 11: Plot of magnetic induction intensity versus time for $a=1$ and $b=1$.

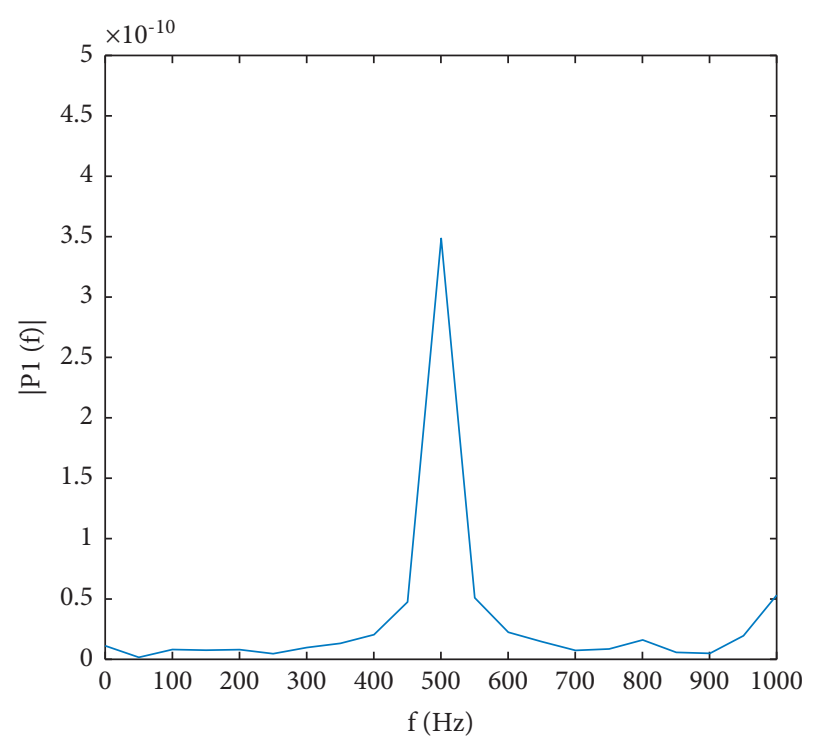

Figure 12: Fourier transform image at $a=1$ and $b=1$. 


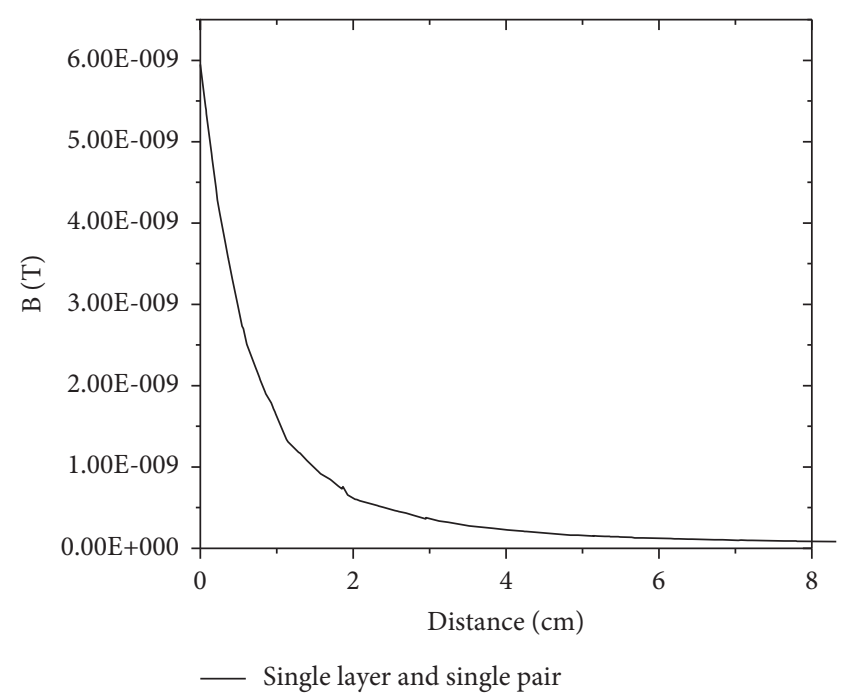

FIgURE 13: Variation of magnetic flux density mode with distance for $a=1$ and $b=1$.

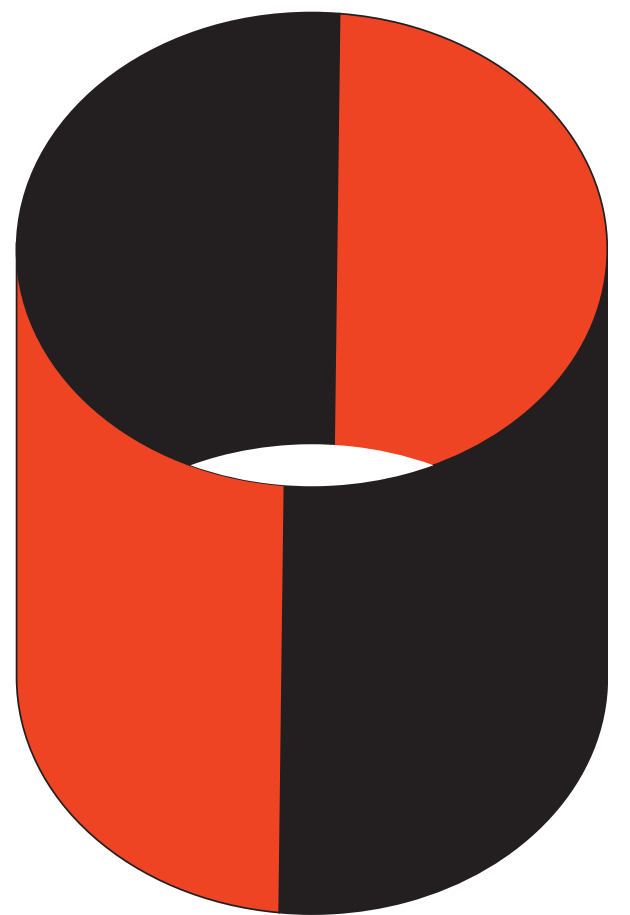

FIGURE 14: Structure of a single-layer two-pair $(a=1, b=2)$ electret thin-film mechanical antenna.

Figure 12, from which we can see that when the electret film $\log a=1$, the frequency of the electromagnetic wave emitted by the antenna is equal to the motor rotation, both at $500 \mathrm{~Hz}$; according to the data obtained from the axis, the image of the variation of magnetic induction intensity $B$ with distance can be obtained as shown in Figure 13, which shows that the maximum value of the magnetic flux density mode $B$ is inversely proportional to the distance $D$.

Keeping all other conditions constant, changing only the value of a to 2 changes the number of electret pairs from a single pair to a double pair, its $3 \mathrm{D}$ model will change to

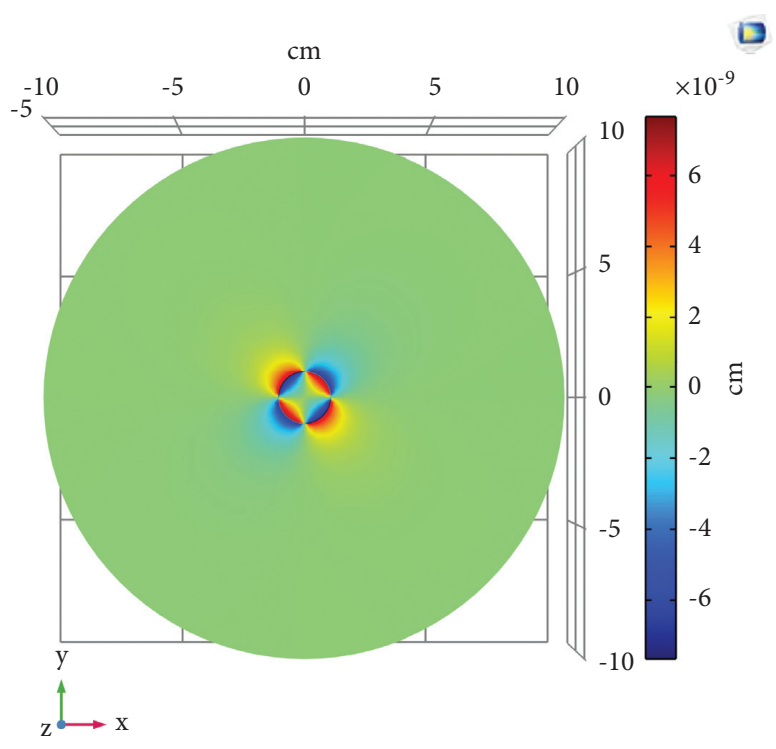

FIGURE 15: Magnetic field cloud at $a=1, b=2$.

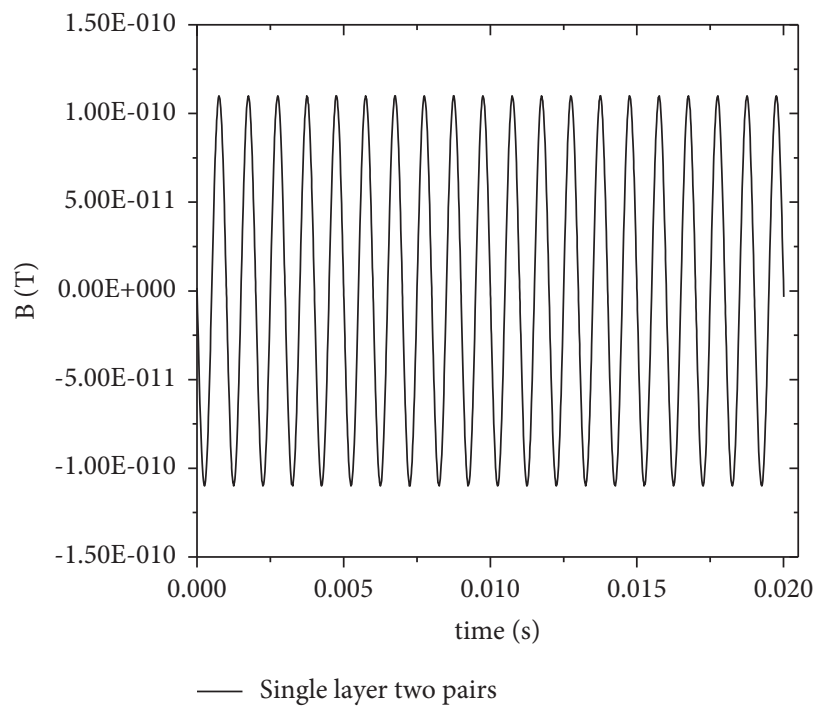

FIgURE 16: Plot of magnetic induction intensity as a function of time for $a=1$ and $b=2$.

Figure 14, as shown in Figure 15, and the simulation yields a magnetic field cloud for the single-layer double pair case, as shown in the diagram, and again from the data obtained at the probe and the axis yields Figures 16 and 17, S11 parameters as shown in Figure 18 can be obtained through calculation, and the E-plane gain and $\mathrm{H}$-plane gain are shown in Figures 19 and 20, respectively.

It can be seen from the figure that after changing the number of electret film pairs $a=2$, the frequency of magnetic induction intensity at the probe increases with time, and by performing the Fourier transform in Figure 16, we can obtain Figure 21, and by observation, it is found that when the number of electret film pairs becomes two pairs, the frequency of the electromagnetic wave emitted by the transmitting antenna changes from $500 \mathrm{~Hz}$ to $1000 \mathrm{~Hz}$, which is twice the rotation frequency of the motor; 


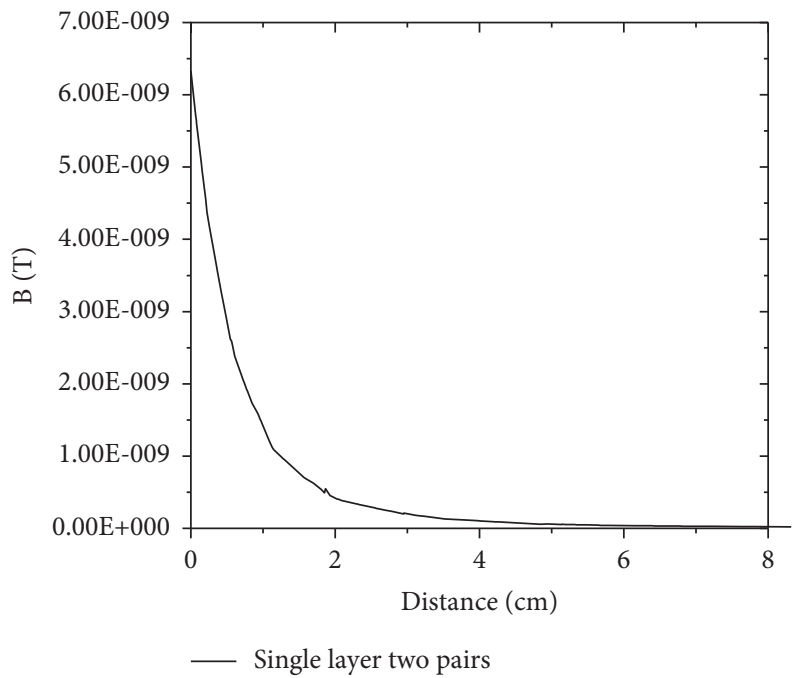

Figure 17: Variation of magnetic flux density mode with distance for $a=1$ and $b=2$.

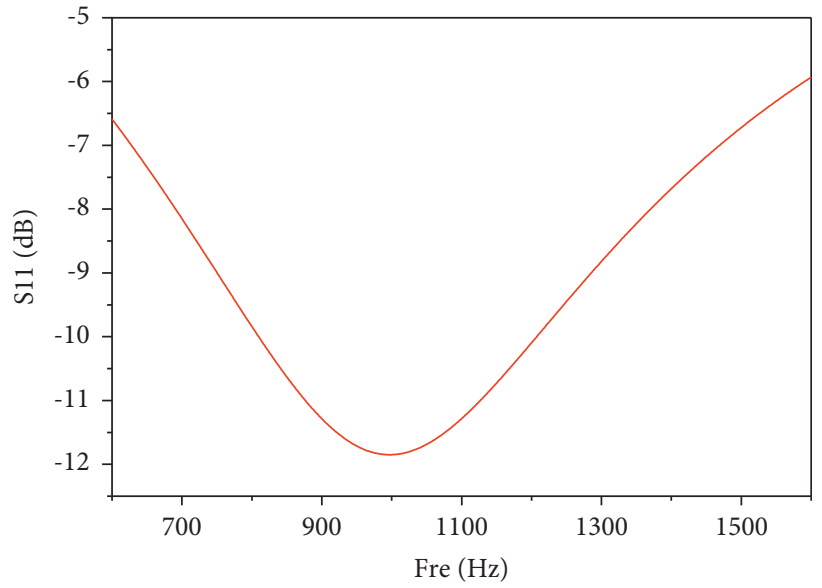

$-\mathrm{B}$

FIGURE 18: S11 parameters for $a=1$ and $b=2$.

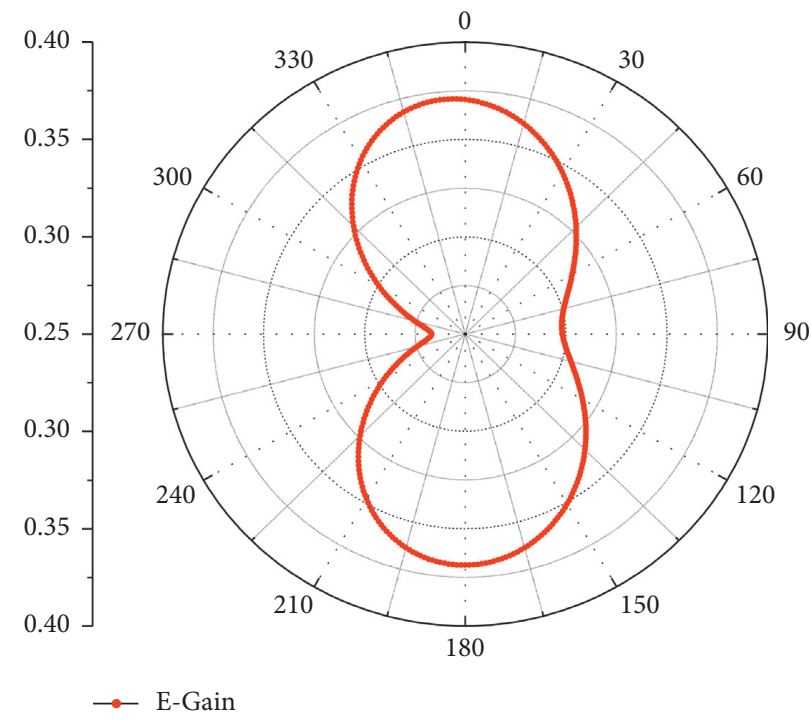

FigURe 19: E-surface gain at $a=1$ and $b=2$. 


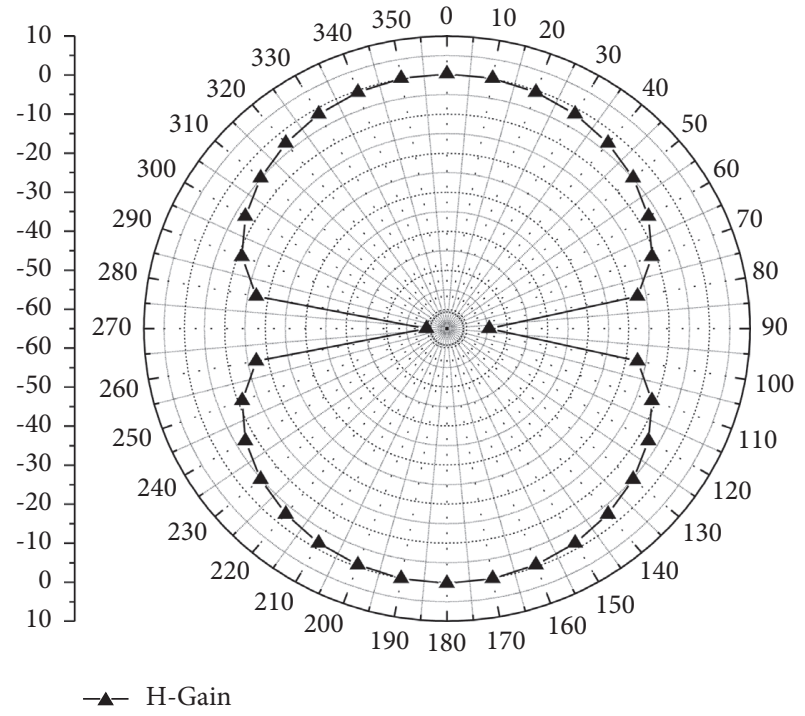

FIgURE 20: H-surface gain at $a=1$ and $b=2$.

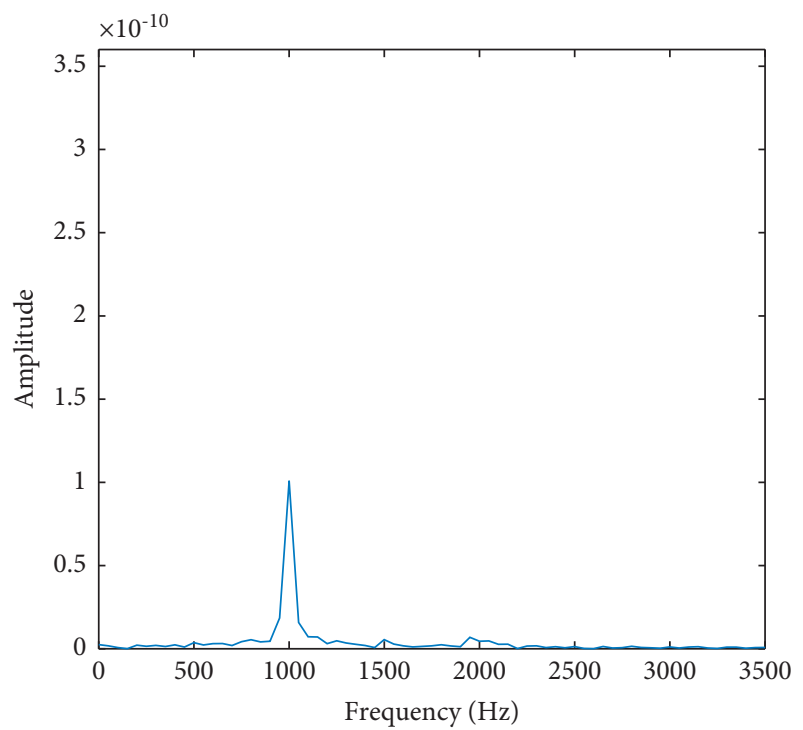

FIgURE 21: Fourier transform image at $a=1, b=2$.

observation of the axes Figure 17 drawn from the data at the axis shows that the maximum value of the magnetic flux density mode amplitude is still inversely proportional to the position $d$ from the transmitting point, but the change of the magnetic flux density mode with distance is not obvious with $a=1$ and $b=1$. We will compare the specific relationship later on.

Based on the analysis of the magnetic field generated by the mechanical antenna for $a=1, b=1 ; a=1, b=2$, we conjecture that the variation of the magnetic field generated by the mechanical antenna will have the same pattern when $a$ is constant and $b=3$ and $b=4$. Therefore, the electret film logarithm was adjusted to $b=3$ and $b=4$, respectively, the model is shown in Figure 22, while keeping other parameters constant, and the following

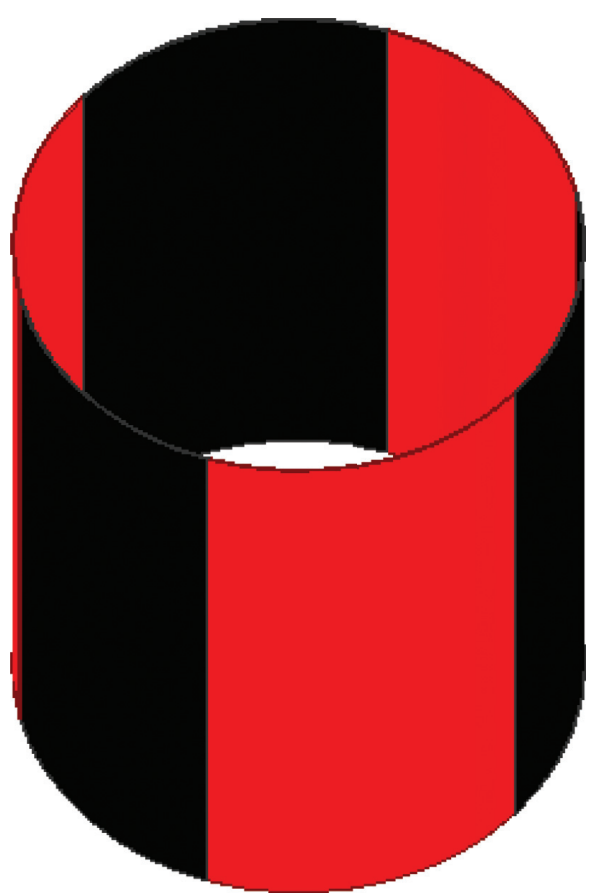

(a)

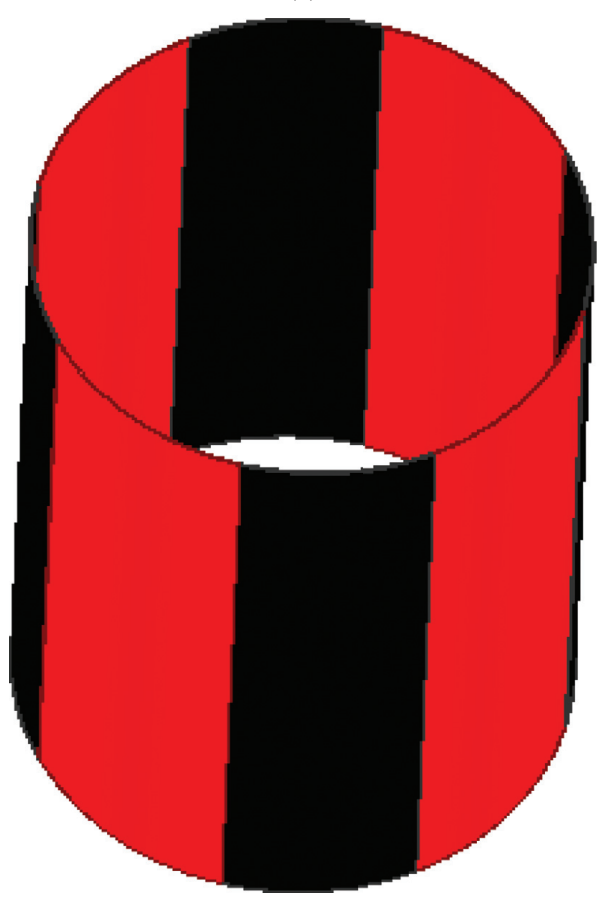

(b)

FIgURE 22: Structure of a single-layer three-pair $(a=1, b=3)$ (a) and single-layer four-pair $(a=1, b=4)$ (b) electret thin-film mechanical antenna.

images were obtained by extracting the data at the probe and the axis, respectively. From the images, it can be seen that our conjecture is confirmed; at the probe, the magnetic induction intensity still varies sinusoidally and periodically with time; at the axis, the maximum value of the magnetic flux density mode amplitude is still inversely proportional to the position $D$ from the emission 


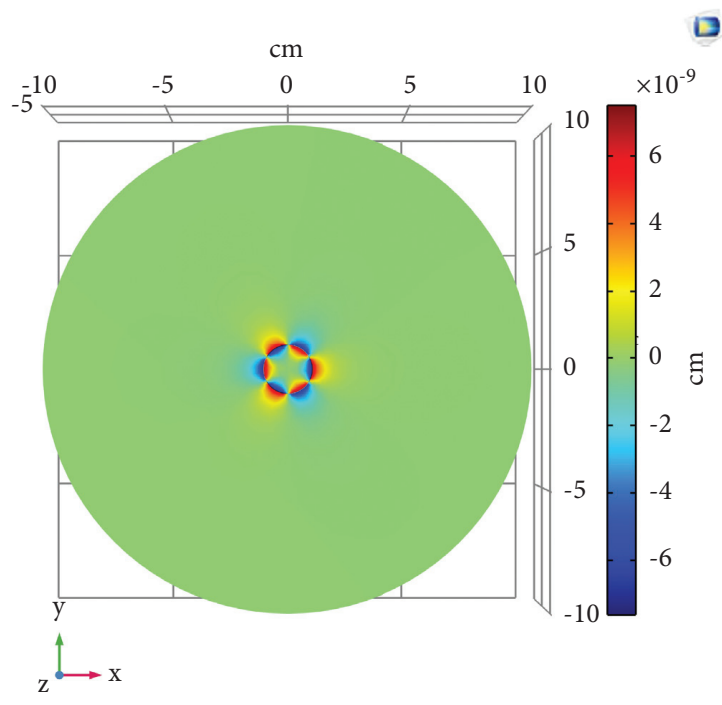

(a)

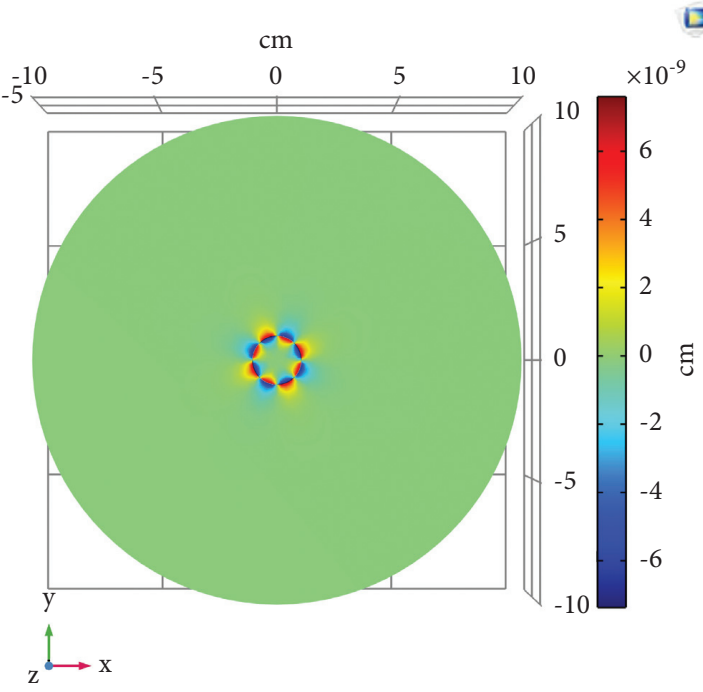

(b)

Figure 23: Magnetic field clouds for $a=1, b=3$ (a) and $a=1, b=4$ (b)

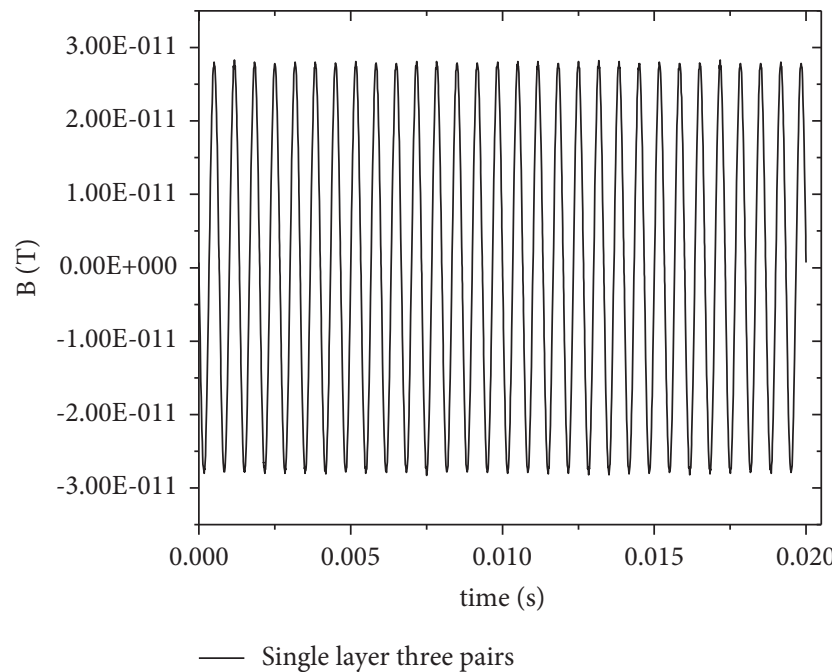

(a)

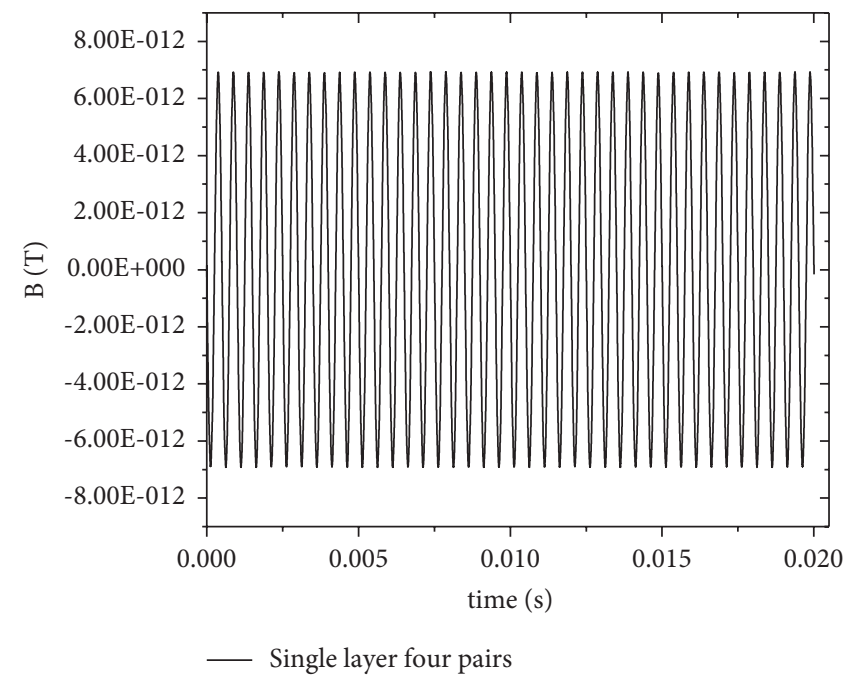

(b)

FIgURe 24: Plot of magnetic induction intensity versus time for a single three-pair $(a=1, b=3)(\mathrm{a})$ and a single four-pair $(a=1, b=4)(\mathrm{b})$.

point. In the following, the electret thin-film mechanical antenna is modeled and analyzed for the two cases of $a=1, b=3$ and $a=1, b=4$. The geometrical models are shown in Figure 22, respectively, and the magnetic field analysis is carried out for the above two geometrical models to obtain the magnetic field clouds for the two cases of $a=1, b=3$ and $a=1, b=4$ as the left and right plots in Figure 23, respectively. Separate Fourier transform of Figure 24 can be obtained from Figure 25; from the Fourier transform diagram, it can be seen that when the electret film pairs are $b=3$ and $b=4$, respectively, the frequency of electromagnetic waves emitted by the transmitting antenna is $1500 \mathrm{~Hz}$ and $2000 \mathrm{~Hz}$, respectively, three times and four times the frequency of motor rotation.

Through COMSOL finite element simulation, S11 parameters, E-plane gain, and s-plane gain at $a=1$ and $b=3$ and 4 as shown in Figures 26-28 can be obtained.

Respectively, $a=1, b=1 ; a=1, b=2 ; a=1, b=3 ; a=1$, $b=4$ when the mechanical antenna at the probe magnetic induction intensity as a function of time is plotted on a graph such as Figure 29; from the graph, it can be clearly seen that, with the increase in the number of electret film $\log b$, the transmitting antenna emitted electromagnetic wave frequency higher and higher, respectively, leading to the four frequencies of the sine curve to do Fourier transform. The 


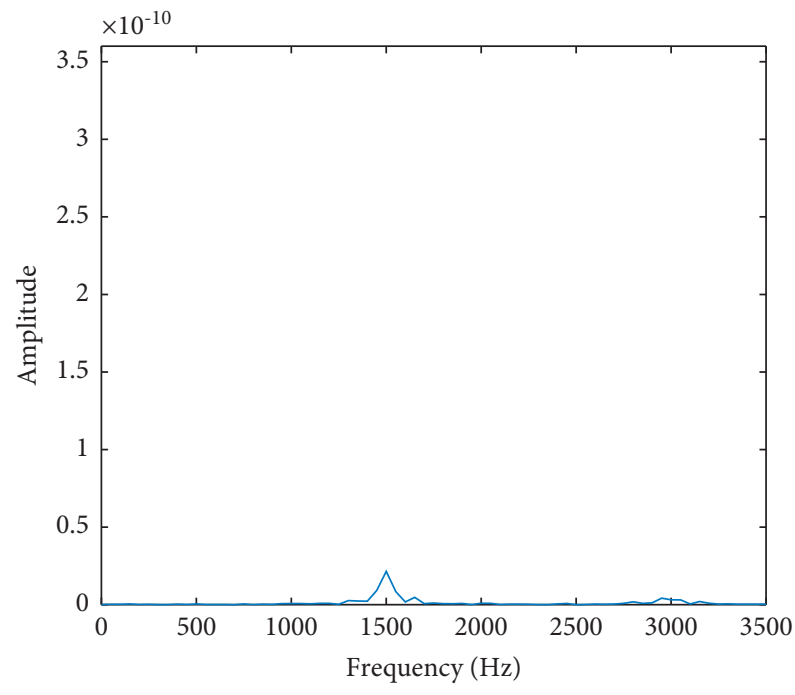

(a)

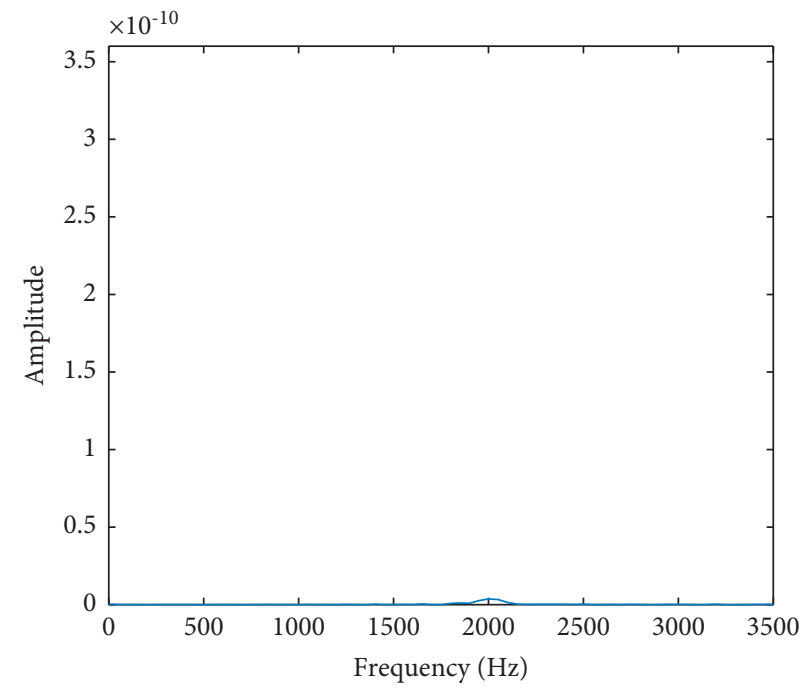

(b)

FiguRe 25: Fourier transform plots for a single three-pair $(a=1, b=3)$ (a) and a single four-pair $(a=1, b=4)(\mathrm{b})$.

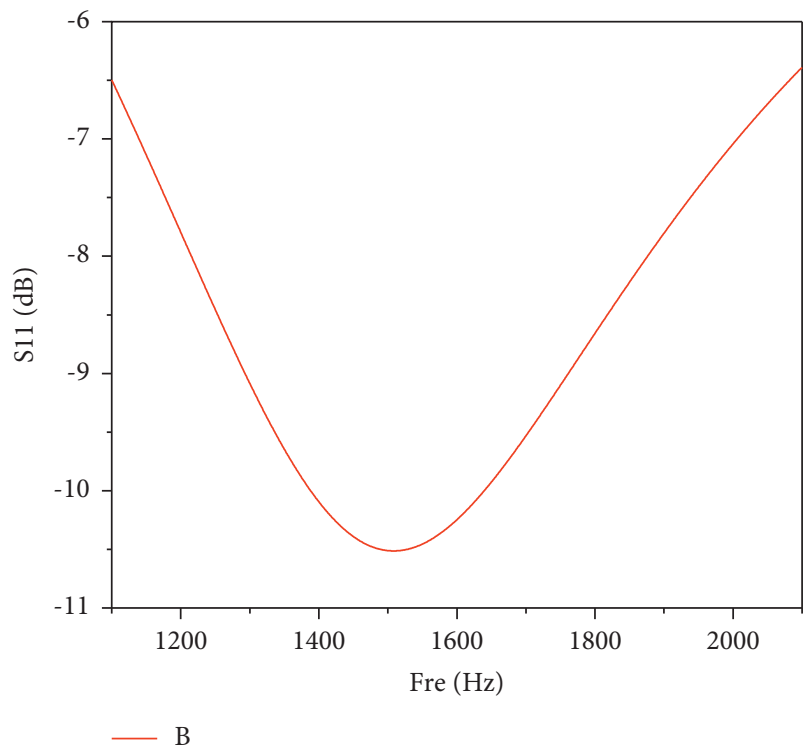

(a)

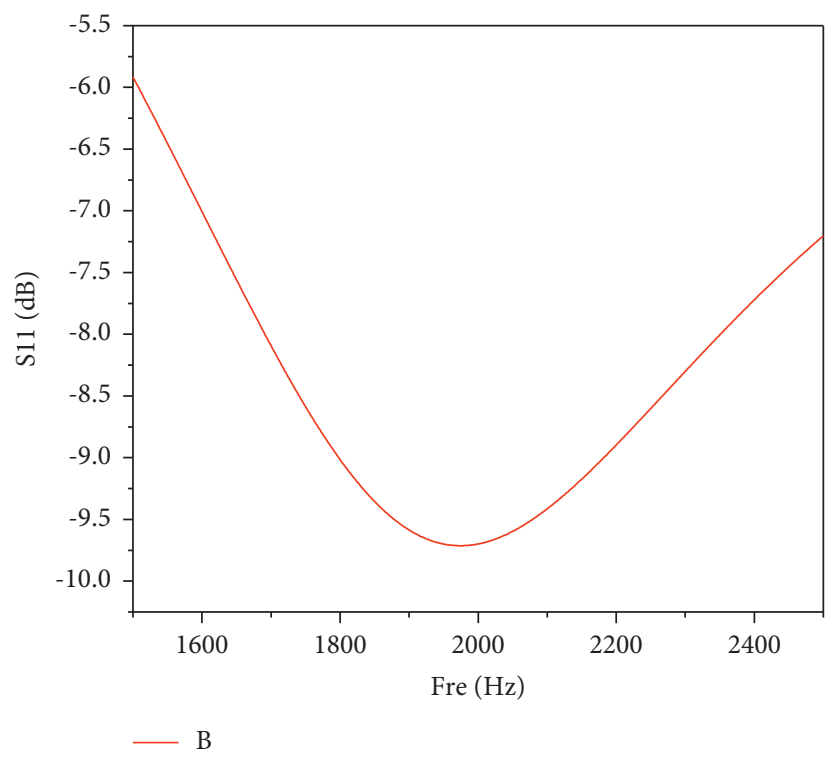

(b)

FIgURE 26: S11 parameters for a single layer of three pairs $(a=1, b=3)$ (a) and a single layer of four pairs $(a=1, b=4)(\mathrm{b})$.

relationship between the number of electret film pairs $b$, the initial frequency $f 0$, and the frequency of the emitted electromagnetic wave can be easily observed in Figure 30, where $b$ is the number of electret film pairs and $f 0$ is the frequency of the emitted electromagnetic wave of the emitting antenna when a single pair of electret films is used, which can also be said to be the frequency of the motor rotation. Figure 31 is difficult to see the relationship between the maximum value of the flux density mode and the distance $d$ when different pairs of electret film. We will change Figures 13, 17, and 31 to a graph to get Figure 32. From Figure 32, it is easy to see that, with the increase in the number of electret pairs, the maximum value of the flux density mode amplitude at the same point decreases, and with the increase in curvature, the maximum value of the flux density amplitude decreases. The rate of decrease of the maximum value increases accordingly as the curvature continues to increase. 


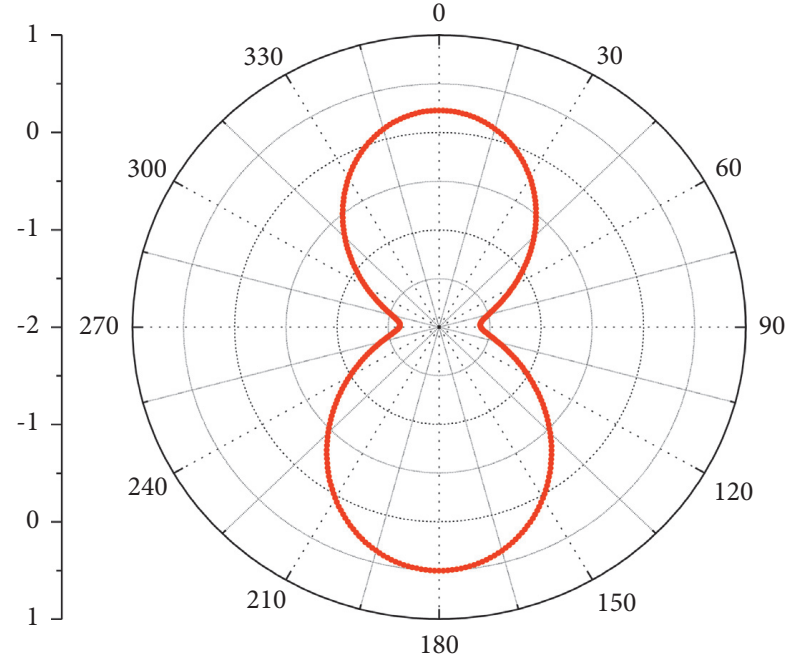

$\rightarrow$ E-Gain

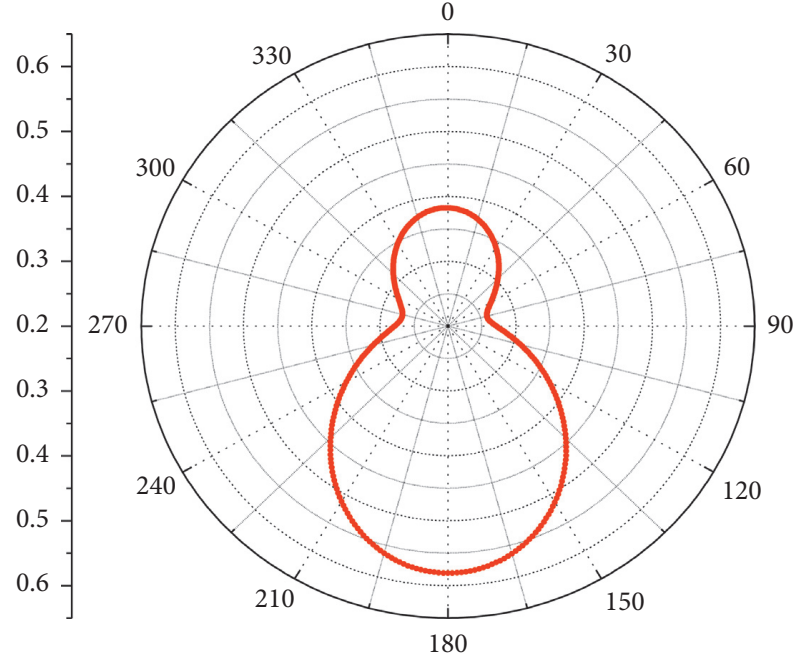

E-Gain

(a)

(b)

Figure 27: E-surface gain diagram for $a=1, b=3$ (a) and $a=1, b=4$ (b).

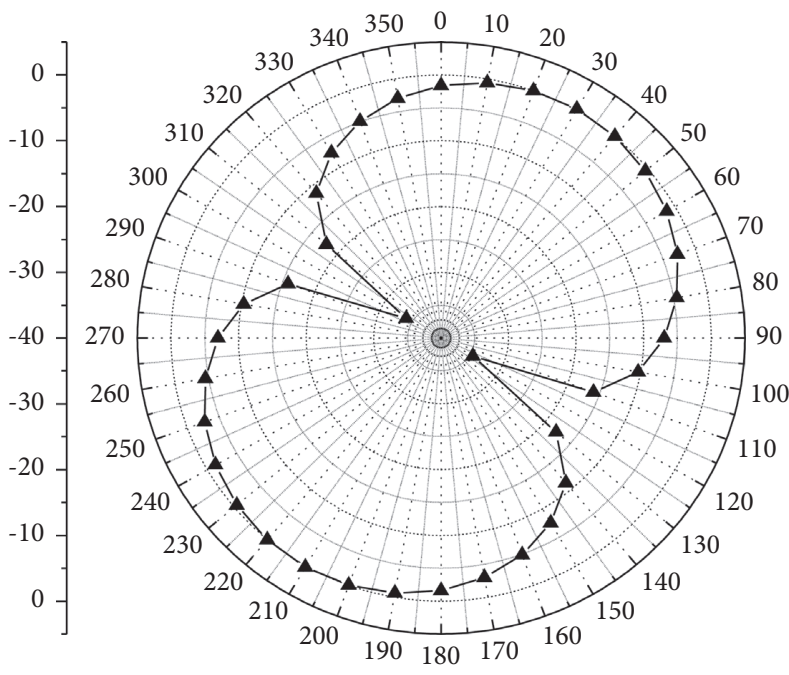

H-Gain

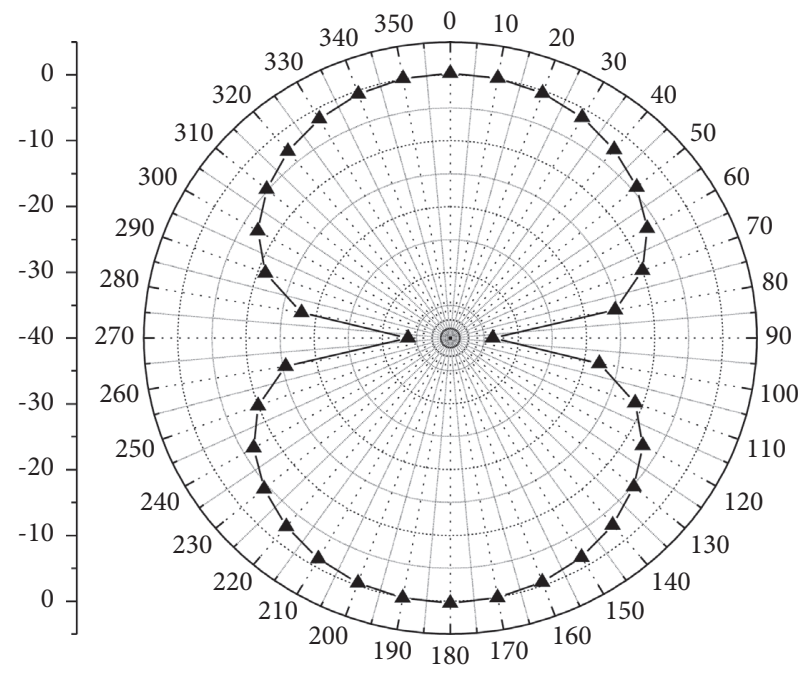

H-Gain

(b)

FIgURE 28: H-plane gain diagram for $a=1, b=3$ (a) and $a=1, b=4$ (b).

\section{Simulation and Analysis of Single-Layer and Multilayer Electret Thin-Film Mechanical Antennas}

The previous subsection analyzed the variation of the magnetic induction $B$ of the mechanical antenna with time as well as the distance $D$ for $a=1$ and $b=1,2,3$, and 4 , respectively. This subsection builds on the previous subsection to analyze the radiation of a multilayer multipair electret thin-film electret antenna. Figure 33 shows a threedimensional model of a two-layer multipair from the first to the fourth from the left, and Figure 34 shows a three-dimensional model of a three-layer multipair from the first to the fourth from the left.

The magnetic field distribution for each of Figures 33 and 34 was analyzed by COMSOL to obtain the following magnetic field distribution for each of the eight pairs of figures. And Figures 35 and 36 show the magnetic field nephogram when $a=2, b=1,2,3,4$ and $a=3, b=1,2,3,4$, respectively.

Through the COMSOL simulation software, the finite element analysis of the above eight models is carried out, 


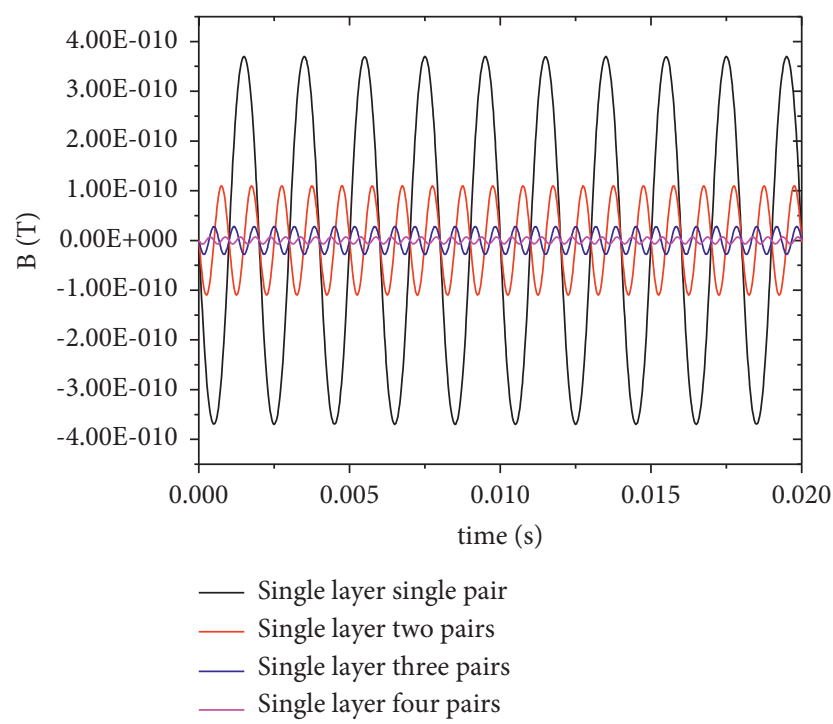

FIgURE 29: Plot of magnetic induction intensity versus time for $a=1 ; b=1, b=2, b=3, b=4$.

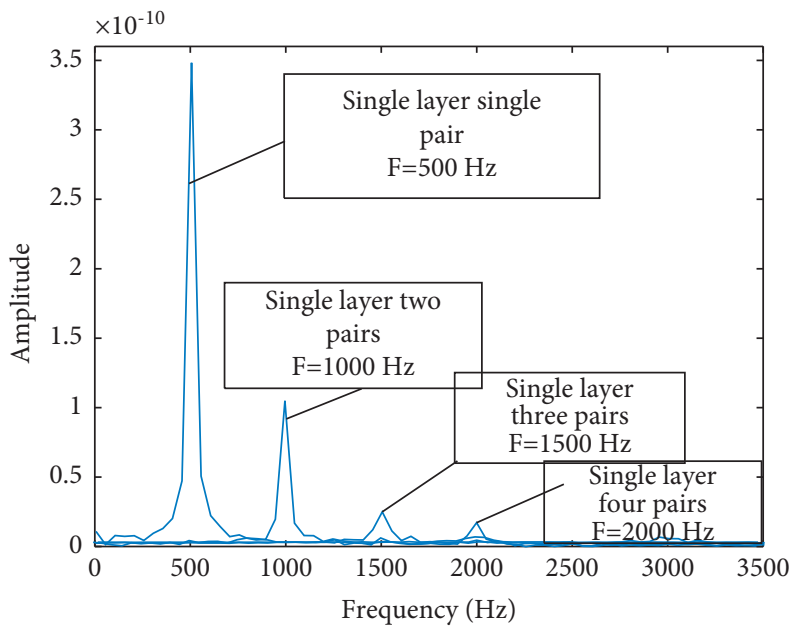

FIGURE 30: Fourier transform plots for $a=1 ; b=1, b=2, b=3, b=4$.

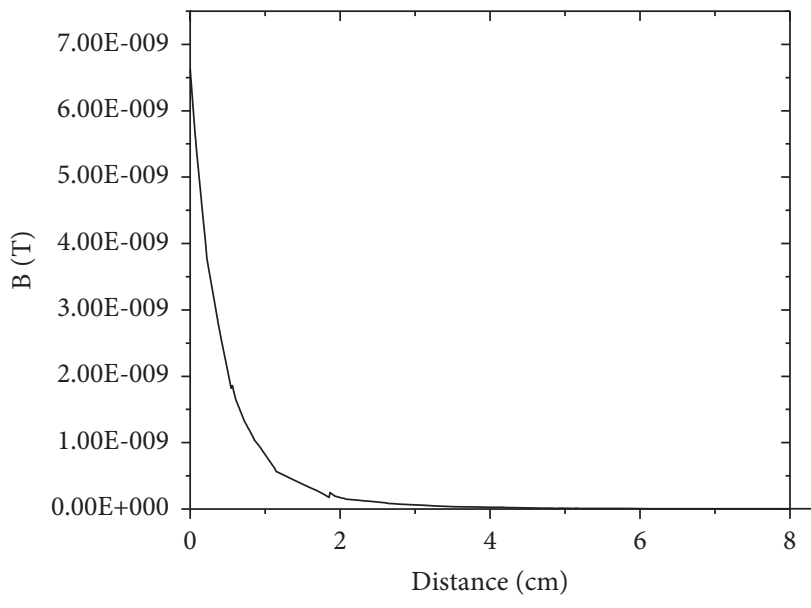

- Single layer three pairs

(a)

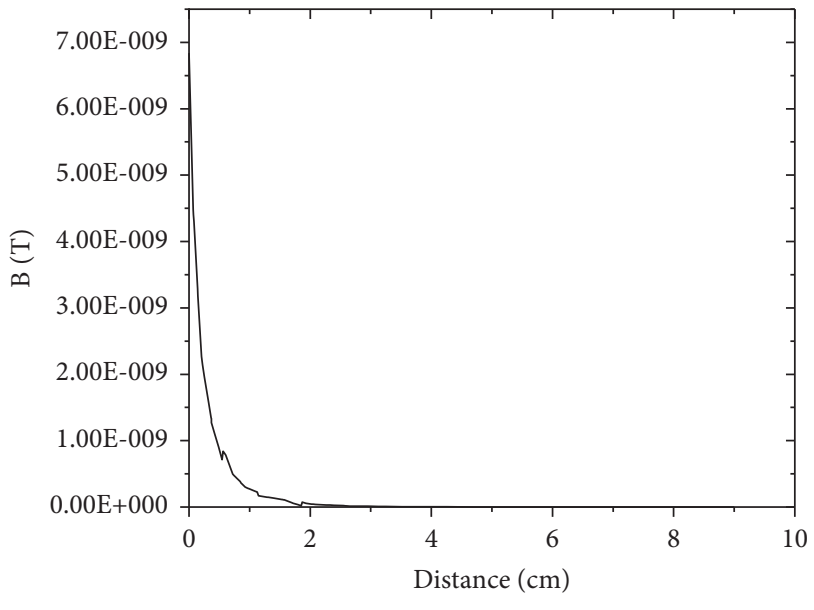

Single layer four pairs

(b)

FIGURE 31: Variation of magnetic flux density mode with distance for a single three-pair $(a=1, b=3)$ (a) and a single four-pair $(a=1, b=4)(b)$. 

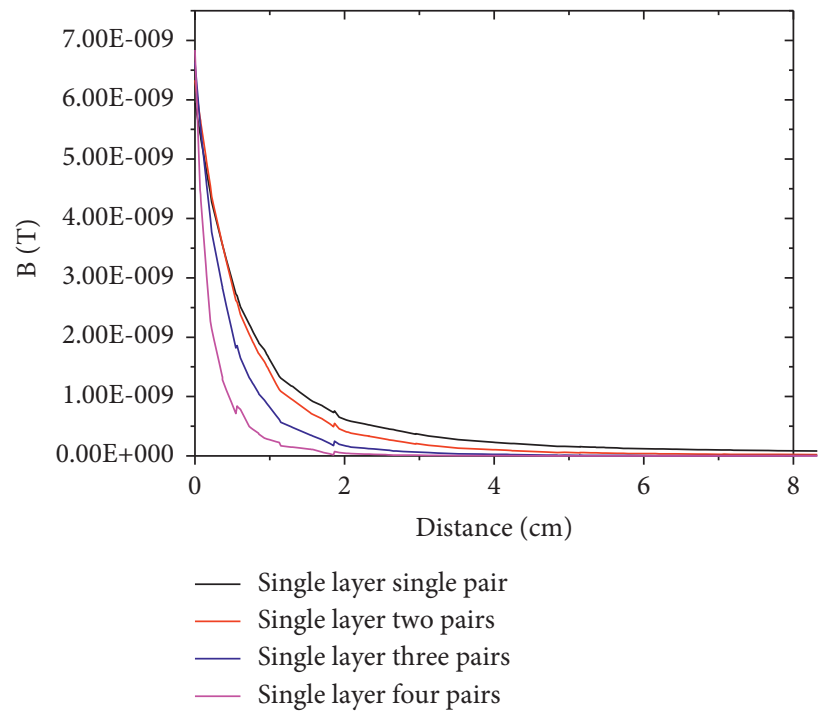

Figure 32: Plot of magnetic flux density mode versus distance for $a=1 ; b=1, b=2, b=3, b=4$.

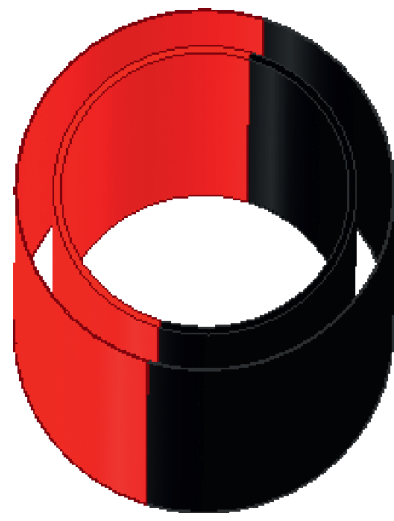

(a)

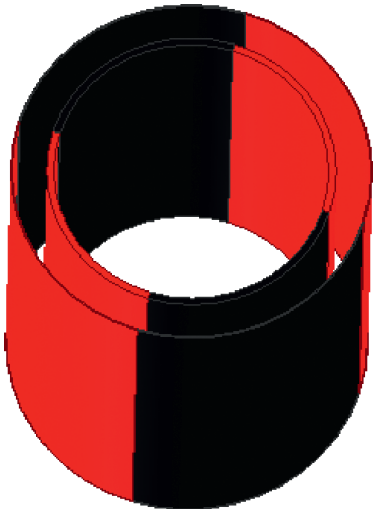

(b)

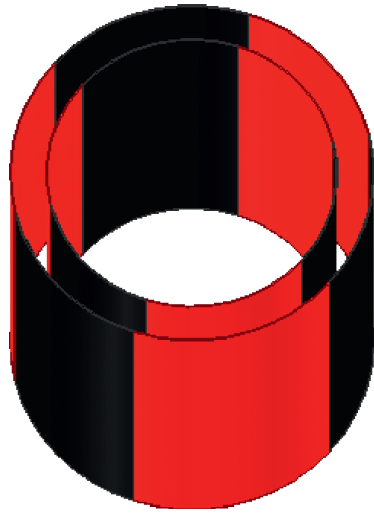

(c)

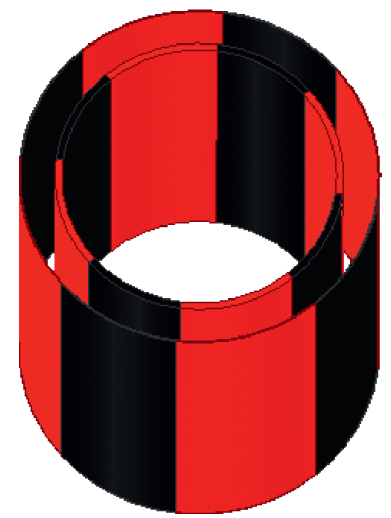

(d)

Figure 33: Three-dimensional model of the mechanical antenna for $a=2, b=1$ (a); $a=2, b=2$ (b); $a=2, b=3$ (c); $a=2, b=4$ (d).

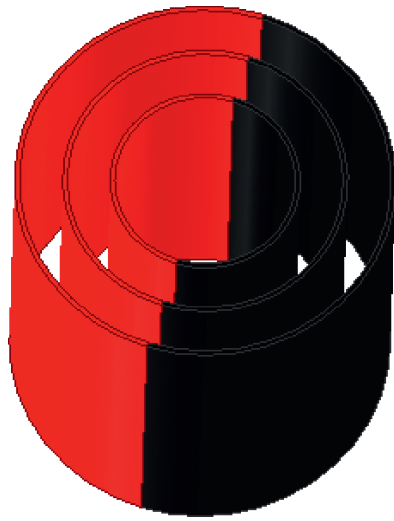

(a)

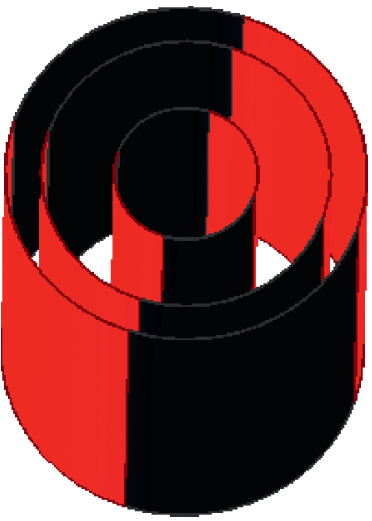

(b)

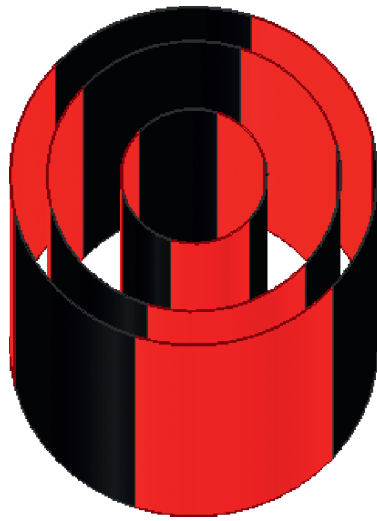

(c)

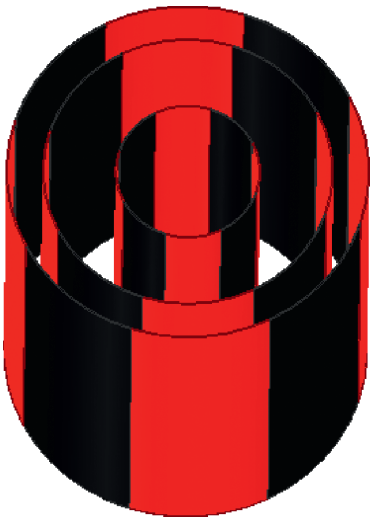

(d)

FIgURE 34: Three-dimensional model of the mechanical antenna for $a=3, b=1$ (a); $a=3, b=2$ (b); $a=3, b=3$ (c); $a=3, b=4$ (d). 


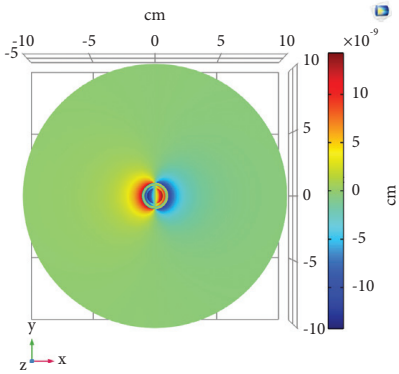

(a)

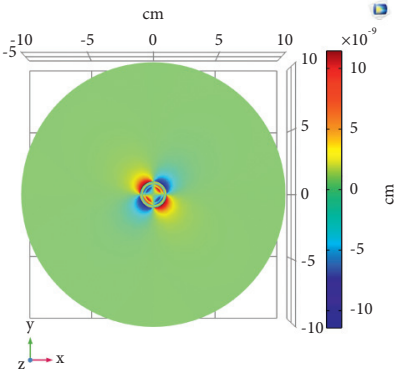

(b)

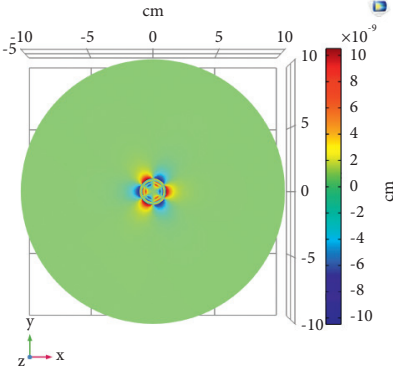

(c)

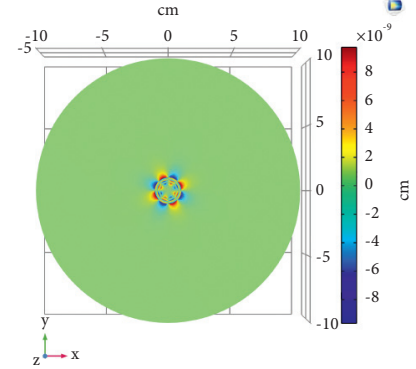

(d)

Figure 35: Magnetic field clouds for $a=2, b=1$ (a); $a=2, b=2$ (b); $a=2, b=3$ (c); $a=2, b=4$ (d).

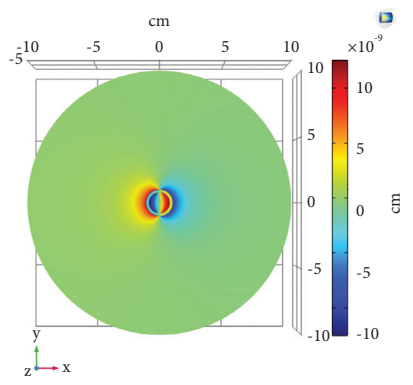

(a)

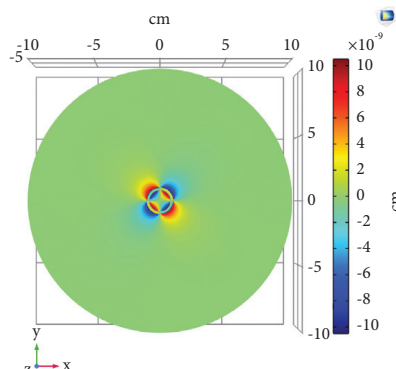

(b)

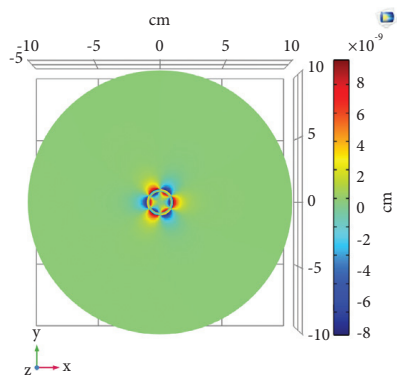

(c)

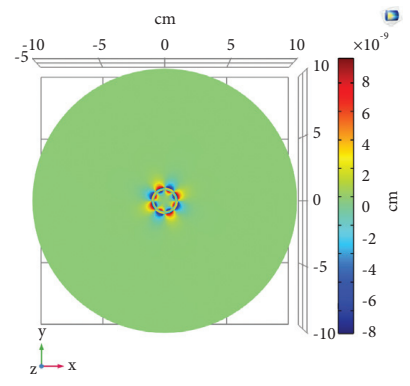

(d)

Figure 36: Magnetic field clouds for $a=3, b=1$ (a); $a=3, b=2$ (b); $a=3, b=3$ (c); $a=3, b=4$ (d).

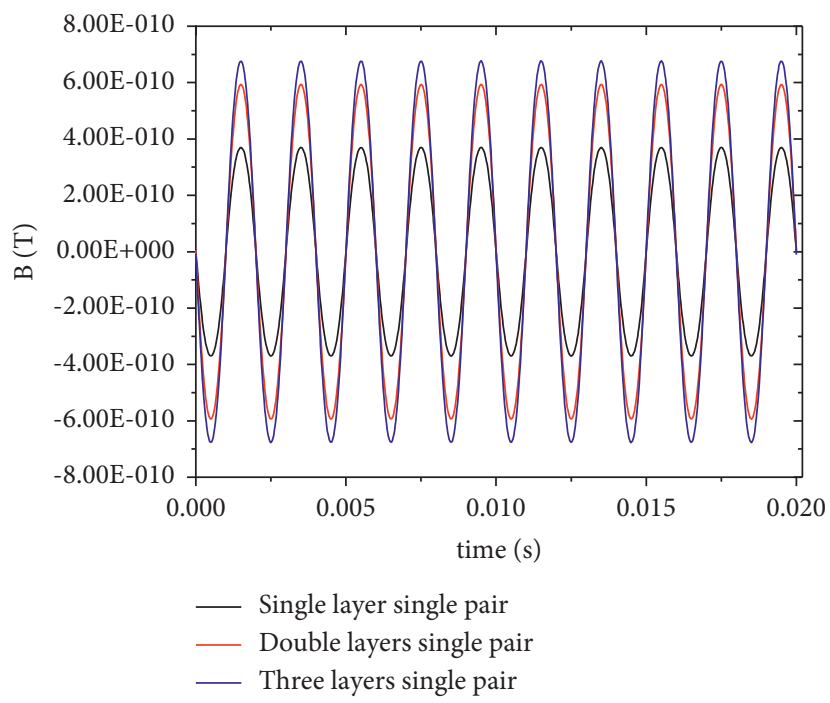

FIgURE 37: Plots of magnetic induction intensity versus time for $a=1, b=1 ; a=2, b=1$; and $a=3, b=1$.

respectively, and the data obtained at the probe and the axis are plotted on a graph, respectively, and Figures 37 and 38 can be obtained. Observing Figure 37, it is easy to see that the frequency of antenna emission is only related to the logarithm $B$ of the electret film but has nothing to do with the layer $a$ of the electret film. It can be seen from Figure 37 and Figure 38 that when $a$ is larger, that is, the number of layers of electret film is more, and $B$ is smaller, that is, the number of pairs of electret film is less, the magnetic induction at the probe is stronger, and the attenuation of magnetic flux density mode is slower.

\section{Attenuation of Electret-Type Mechanical Antennas in Different Media}

If the properties of the medium are changed and the frequency is kept constant, Figure 39 plots the variation curve of magnetic induction $\mathrm{B}$ with distance in three different 


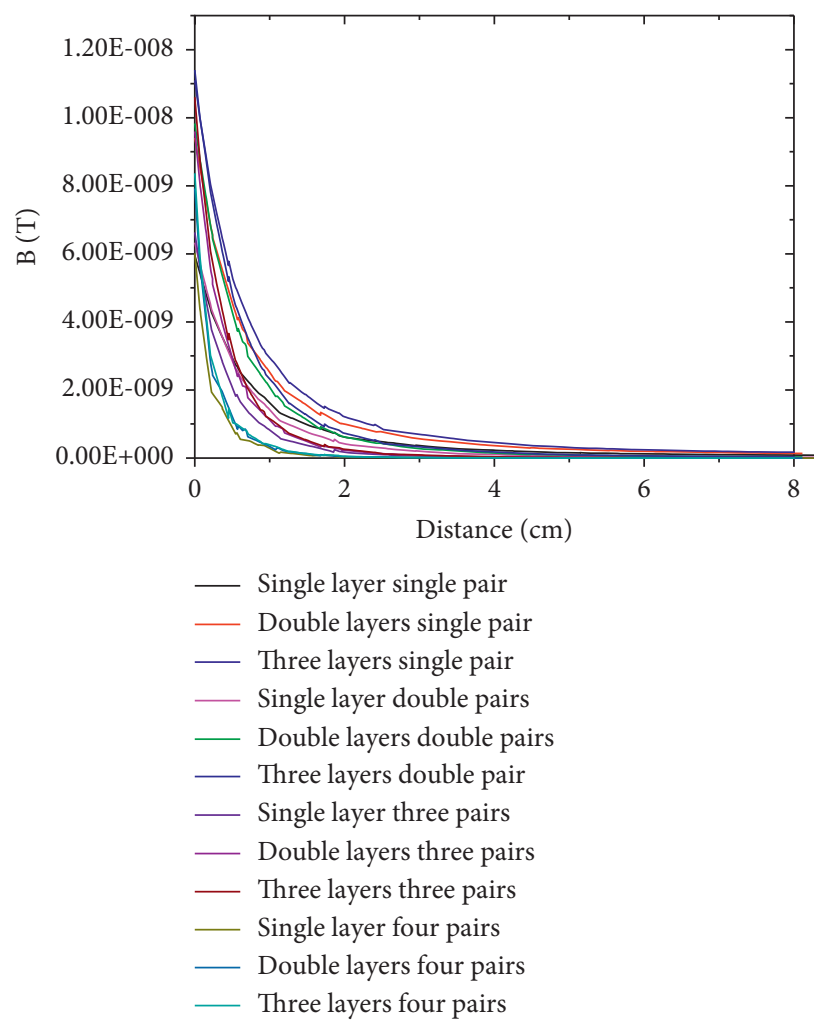

Figure 38: Plots of magnetic flux density modes with distance for $a=1,2,3, b=1 ; a=1,2,3, b=2 ; a=1,2,3, b=3 ; a=1,2,3, b=4$.

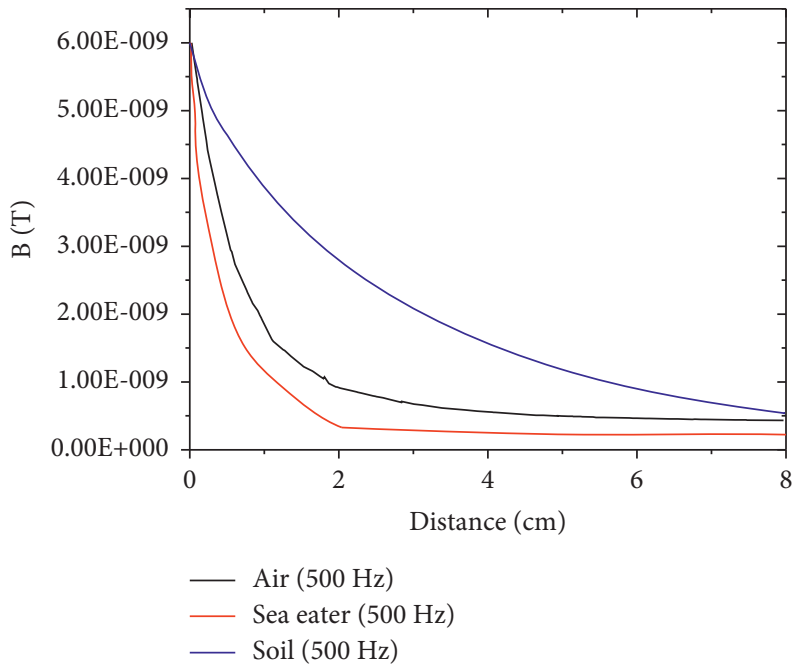

FIgURe 39: Magnetic induction as a function of distance for $a=1$ and $b=1(500 \mathrm{~Hz})$ in different media.

media, air, seawater, and soil, for the cases $a=1$ and $b=1$. Here, the relative permittivity of soil is 4 , and the conductivity is $0.015 \mathrm{~S} / \mathrm{m}$; the relative permittivity of seawater is 81 , and the conductivity is $4 \mathrm{~S} / \mathrm{m}$. It can be seen that the magnetic induction intensity decays violently in seawater but relatively slowly in air and soil. When used in seawater, the magnetic induction can be increased by increasing the number of layers of the electret film, but it is also important to avoid increasing the number of electret film pairs too much to avoid increasing the emission frequency and thus causing the magnetic induction to decay too quickly in seawater.

\section{Conclusions}

Through comsol modeling and analysis, it can be concluded that the increase of the number of electret pairs will inevitably increase the emission frequency of the mechanical antenna and make the emission frequency proportional to the number of electret pairs and, at the same time, lead to the decrease of the 
magnetic flux density mode, i.e., the larger $b$ is, the higher the radiation frequency of the mechanical antenna is, the lower the magnetic flux density is, the faster the corresponding magnetic field strength decays, and the shorter the propagation distance is. In order to improve the magnetic induction strength, we can increase the number of layers of electret film, that is, the larger $a$, the greater the magnetic induction strength, in the mechanical antenna volume making the range can moderately increase the value of $a$ in exchange for the increase in magnetic induction strength. To sum up, the mechanical antenna electret film layer and the number of pairs should be selected according to the actual transmission efficiency and transmission distance, in order to realize the good performance of the mechanical antenna in frequency and distance. The electret-type mechanical antenna is several orders of magnitude smaller than the traditional lowfrequency transmitting antenna, which has better concealment in wartime, and at the same time, the electret-type mechanical antenna can be designed as a wearable device, which brings great convenience to the relevant staff; by changing the number of layers and logarithms of the electret film to change the charge distribution mode, it brings the great possibility of changing the radiation frequency of the mechanical antenna and improving the propagation distance. This has opened up great possibilities for research into mine communication, submarine communication, and wearable low-frequency transmitting antennas.

\section{Data Availability}

The basic data used in this study can be found on the following website: https://www.aviso.altimetry.fr/en/home. html.

\section{Conflicts of Interest}

All the data presented in this article are agreed upon and recognized by all the authors, and there are no conflicts of interest between the authors.

\section{Acknowledgments}

This paper was supported by the National Natural Science Foundation of China (61801172). At the same time, the authors would like to thank Professor Niu Youtian and Professor Liu Weina for their help in the simulation part of the paper. At the same time, the authors would like to thank the mechanical antenna research group for their strong support.

\section{References}

[1] H. P. Korner, "Dipole antenna for a portable communication device," US Patent No. 11/738708, 2018.

[2] H. Ding, "DARPA mechanical antenna project may revolutionize military communications," Modern Military, vol. 4, no. 04, pp. 71-73, 2017.

[3] A. K. Mark, F. Matt, H. Andy et al., "A high Q piezoelectric resonator as a portable VLF transmitter," Nature Communications, vol. 10, no. 5, pp. 044905-044983, 2019.

[4] Y. Cui, C. Wang, and X. Song, "A simulation modeling-based method for evaluating the performance of rotating electret- type mechanical antennas:” CN Patnet No. 201910221622.X, 2019.

[5] H. Zheng, J. Zhao, and B. Xiang, "Parallel plate VLF mechanical antenna," in Proceedings of the 2018 IEEE International Symposium on Antennas and Propagation \& USNC/ URSI National Radio Science Meeting, IEEE, Boston, MA, USA, July 2018.

[6] D. J. Zhang, Research on the Mechanism and Modulation Method of Ultra-low Frequency Mechanical Antenna, Xi'an University of Technology, Xi'an, China, 2019.

[7] C. Wang, Y. Cui, and M. Wei, "Mechanically-rotating electret ULF/VLF antenna transmitter," in Proceedings of the 2019 IEEE International Symposium on Antennas and Propagation and USNC-URSI Radio Science Meeting, IEEE, Atlanta, GA, USA, July 2019.

[8] L. C. Zhang, G. J. Chen, X. Hui-Ming, C. Ben-Xiao, H. Huang, and $\mathrm{L}$. Wu, "Preparation of millimeter-scale gate type electric field distribution FEP thin film electret and its charge storage performance," Journal of Physics, vol. 64, no. 23, pp. 311-318, 2015.

[9] G. J. Chen, C. P. Rao, H. Huang, and Y. H. Zhao, "Charge storage properties of interfacially polarized polypropylene thin film electret," Journal of Physics, vol. 64, no. 23, pp. 319-324, 2015

[10] N. Wu, X. Cheng, Q. Zhong et al., "Cellular polypropylene piezoelectret for human body energy harvesting and health monitoring," Advanced Functional Materials, vol. 25, no. 30, pp. 4788-4794, 2015.

[11] W. B. Bin, J. Zhong, Q. Zhong et al., "Surface charge selfrecovering electret film for wearable energy conversion in a harsh environment," Energy \& Environmental Science: Ecotoxicology and Environmental Safety, vol. 9, no. 10, pp. 3085-3091, 2016.

[12] Y. Chu, J. Zhong, H. Liu et al., "Human pulse diagnosis for medical assessments using a wearable piezoelectret sensing system," Advanced Functional Materials, vol. 28, no. 40, 2018.

[13] C. Wang, Y. Cui, and X. Song, "Electret material-based magnetic field propagation model for mechanical antennabased low frequency/very low frequency communications," Journal of Physics, vol. 69, no. 15, 9 pages, 2021.

[14] Y. Cui, C. Wang, X. Song, and B. W. Liang, "Simulation study of mechanical antenna-based low-frequency communication system based on electret material," Journal of Automation, vol. 3, no. 4, pp. 1-8, 2021. 